Hydroécol. Appl. (1989) 1/2, pp. 53-83

\title{
Effets de l'échauffement artificiel de l'eau du Rhône sur le développement et la production des Gammaridae
}

\author{
M.C. Roger et B. Faessel \\ CEMAGREF, Division QEPP, 3, quai Chauveau, 69336 Lyon Cédex 09.
}

Résumé - L'effet d'un réchauffement artificiel de l'eau sur le cycle de développement et la production des Gammaridae est étudié sur 3 stations du Rhône au niveau du Centre de Production Nucléaire du Bugey de novembre 1987 à novembre 1988.

L'examen de l'évolution des effectifs moyens met en évidence une période de naissance à partir de mars suivie d'une phase de croissance. Au niveau de la station réchauffée le recrutement apparaît plus important, favorisé par une température plus élevée.

Lorsque la température atteint $20^{\circ} \mathrm{C}$, en juin sur la station réchauffée, en août sur les deux autres, les effectifs diminuent. De plus, sur le secteur réchauffé, les gammares disparaissent totalement en août quand la température moyenne devient supérieure à $25{ }^{\circ} \mathrm{C}$.

L'étude des histogrammes longueur-fréquence montre que l'élévation de la température de l'eau n'entraîne pas de décalage notable du cycle de développement des gammares. Les estimations de production annuelles ne révèlent pas d'effet global du réchauffement. De même le taux annuel de renouvellement de la biomasse $(P / B)$ ne diffère pas significativement sur les trois sites d'étude.

Summary - From November 1987 to November 1988 observations on three stations of the Rhône were carried out to study the effect on Gammaridae's life history and production of the rise in temperature caused by the cooling water from the Nuclear Power Plant in Bugey (France).

The increase in the number of gammares since march corresponds to a period of birth especially on the heated area of the river where the recruitment is more important because of a higher temperature. During the following months, until June-July the organisms are growing.

When the temperature reaches $20^{\circ} \mathrm{C}$, in June on the heated station, in August on the ambient stations the densite decreases, and on the heated station the gammares disappear completely during August when the temperature exceeds $25^{\circ} \mathrm{C}$. 
The size frequency distribution of the gammares on the three stations shows that the increasing temperature of the water does not induce a notable displacing of the life cycle of this organisms.

The rise in water temperature has no effect on the annual production. Moreover, the ratio of the annual renewing biomass $(P / B)$ does not differ significantly in the three sampling sites.

\section{Sommaire}

\section{Introduction}

\section{Présentation de l'étude}

III. Les Gammares

III.1 Morphologie

III.2 Biologie

\section{Matériel et méthodes}

IV.1 Choix des stations

IV.2 Echantillonnage

IV.3 Techniques de laboratoire

IV.4 Etudes préliminaires

IV.5 Estimation de production

\section{Résultats et interprétation}

V.1 Dynamique des populations

V.2 Cycle de développement

V.3 Estimation de production

\section{Conclusion}

\section{Bibliographie}

\section{Introduction}

Dans tous les systèmes aquatiques, les invertébrés se développent suivant un cycle biologique bien défini, où certaines composantes du milieu jouent un rôle prépondérant, en particulier la température de l'eau, dont dépend étroitement le métabolisme de ces organismes.

La présente étude a pour but de déterminer dans quelle mesure l'élévation de la température de l'eau du Rhône à l'aval du Centre de Production Nucléaire du Bugey a une incidence sur le cycle de développement et la production des Gammaridae.

\section{Présentation du site de l'étude}

\section{Les centrales thermiques.}

Le centre de Production Nucléaire du Bugey est installée à $35 \mathrm{~km}$ de Lyon sur la rive droite du Rhône, en amont de la confluence de l'Ain (Fig. 1).

II comporte cinq tranches, dont les caractéristiques sont portées sur le tableau 1.

Le fonctionnement des centrales induit une modification importante des conditions thermiques. Durant la période d'étude, l'élévation de température, mesurée sur le site de Loyettes soit $6 \mathrm{~km}$ à l'aval, est de $6^{\circ} \mathrm{C}$ en moyenne et peut atteindre $10^{\circ} \mathrm{C}$ en hiver en rive droite (Fig. 2). Jusqu'à la confluence de l'Ain, le réchauffe- 


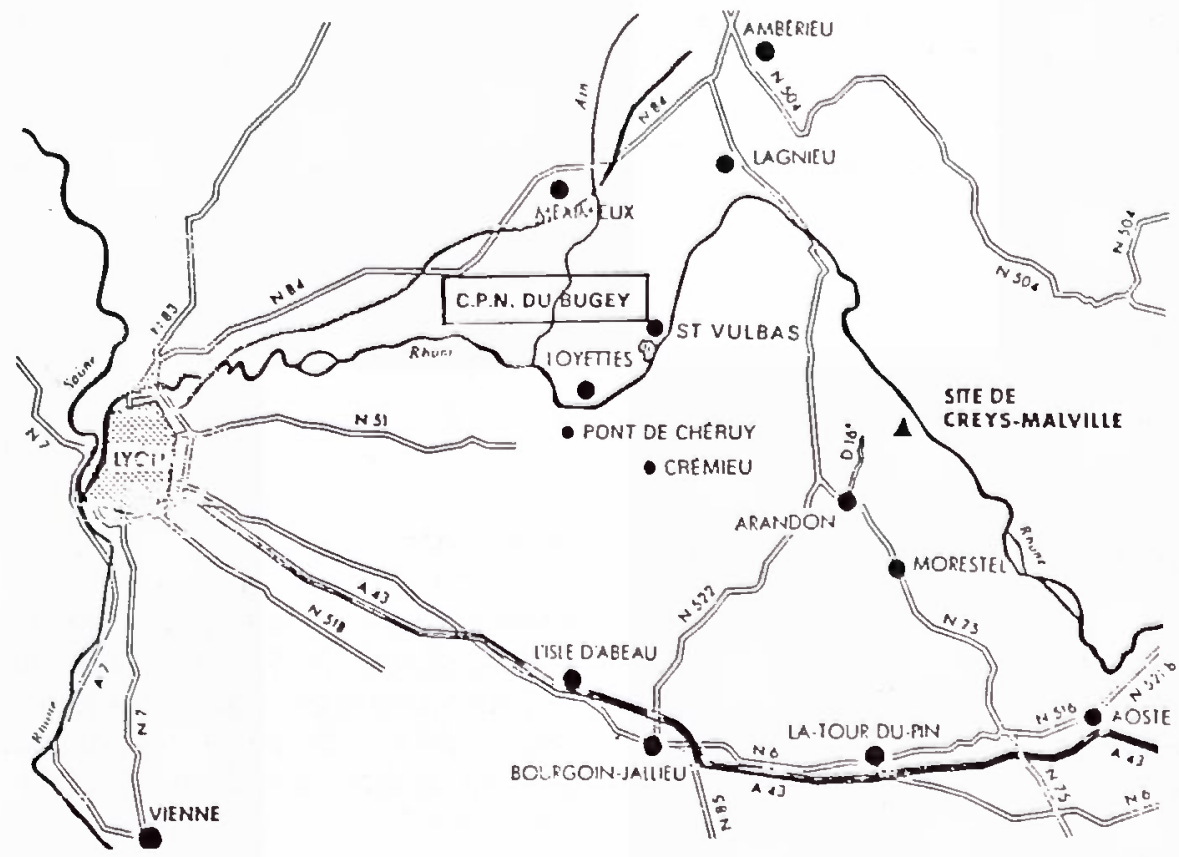

Fig. 1. Plan de situation (1).

ment est beaucoup plus marqué sur cette rive du fleuve qu'en rive gauche.

\section{Hydrologie}

Dans ce secteur le Rhône est soumis à un régime glaciaire à influence pluviale.

Le débit annuel moyen est de $445 \mathrm{~m}^{3} / \mathrm{s}$ avec des maxima en début d'été et un étiage en automne-hiver.

Au niveau du site du Bugey, ce cycle hydrologique naturel est fortement modifié par la gestion des barrages hydroélectriques situés à l'amont, provoquant une succession rapide d'importantes variations de débit.
L'année d'étude est caractérisée par un débit moyen de $560 \mathrm{~m}^{3} / \mathrm{s}$, des crues relativement importantes en janvier, mars et surtout octobre où le débit atteint $1700 \mathrm{~m}^{3} / \mathrm{s}$ (Fig. 3). L'étiage a eu lieu en septembre, octobre avec un débit inférieur à $200 \mathrm{~m}^{3} / \mathrm{s}$.

\section{Qualité physico-chimique}

La conductivité observée sur ce secteur (tableau 2) traduit une forte minéralisation qui, associée à une alcalinité voisine de $200 \mathrm{mg} / \mathrm{l}$, permet de qualifier les eaux de productives.

En ce qui concerne les composés azotés, la présence non négligeable d'azote ammoniacal, et de nitrites,

(1) D'après document EDF 1976 : Le Centre de Production Nucléaire du Bugey. 
Tableau 1. Caractéristiques des différentes tranches nucléaires implantées sur le site du Bugey.

\begin{tabular}{|c|c|c|c|c|}
\hline Tranche & Puissance & $\begin{array}{c}\text { Type de circuit } \\
\text { de refroidissement }\end{array}$ & Débit prélevé & $\begin{array}{c}\text { Date de mise } \\
\text { en fonctionnement }\end{array}$ \\
\hline 1 & $540 \mathrm{MWe}$ & ouvert & $31 \mathrm{~m}^{3} / \mathrm{s}$ & 1972 \\
2 & $925 \mathrm{MWe}$ & ouvert & $42,5 \mathrm{~m}^{3} / \mathrm{s}$ & 1978 \\
3 & $925 \mathrm{MWe}$ & ouvert & $42,5 \mathrm{~m}^{3} / \mathrm{s}$ & 1978 \\
4 & $900 \mathrm{MWe}$ & fermé & $1 \mathrm{~m} / \mathrm{s}$ & 1979 \\
5 & $900 \mathrm{MWe}$ & fermé & $1 \mathrm{~m}^{3} / \mathrm{s}$ & 1979 \\
\hline Total & $4190 \mathrm{MWe}$ & & $118 \mathrm{~m}^{3} / \mathrm{s}$ & \\
\hline
\end{tabular}

correspond à un état critique de pollution organique (Nisbet, Verneaux, 1970).

De même, les teneurs en sulfates et phosphates, sans être excessives, témoignent de l'arrivée d'effluents urbains et/ou industriels et sont susceptibles d'entraîner une dégradation sensible du milieu.

\section{Régime thermique}

A l'amont du site du Bugey, le régime thermique du Rhône présente en moyenne une amplitude annuelle voisine de $20^{\circ} \mathrm{C}$. Durant la période d'étude les écarts varient de $5,2^{\circ} \mathrm{C}$ en février à $23,5^{\circ} \mathrm{C}$ en août (Fig. 4).

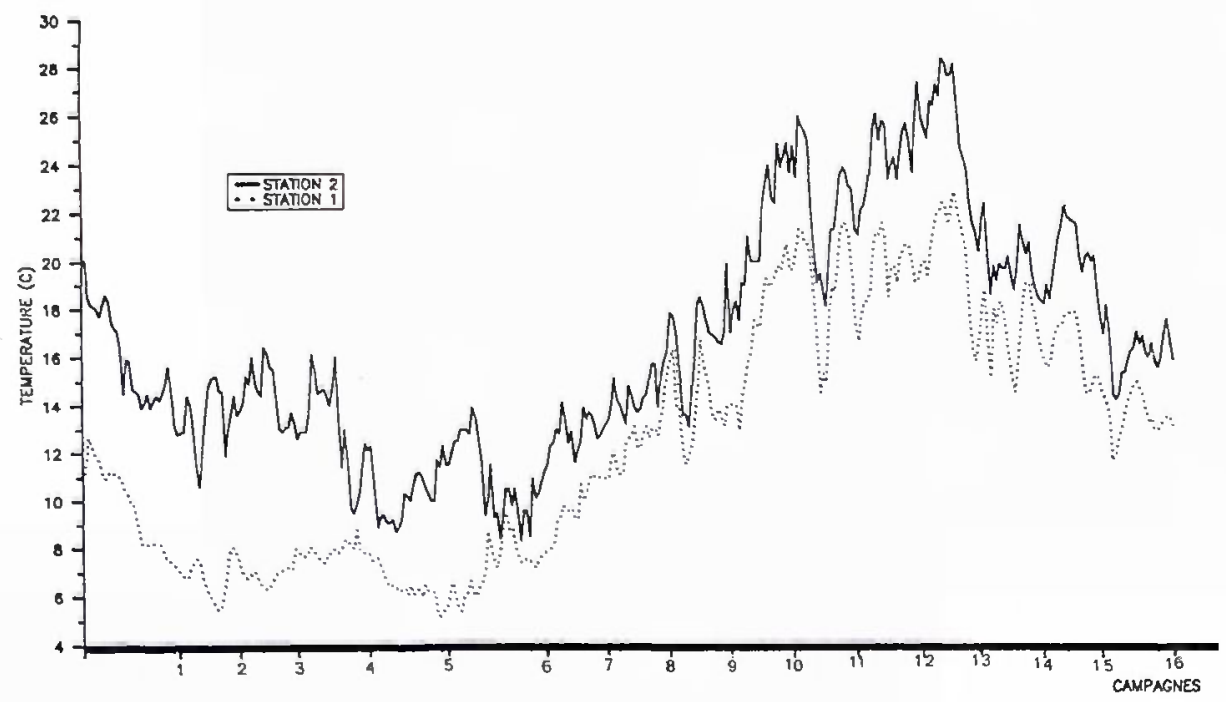

Fig. 2. Températures moyennes journalières relevées aux stations amont (1) et aval en rive droite (2) de novembre 1987 à novembre 1988. Les nombres de 1 à 16 localisent les campagnes de mesures dans le temps. 


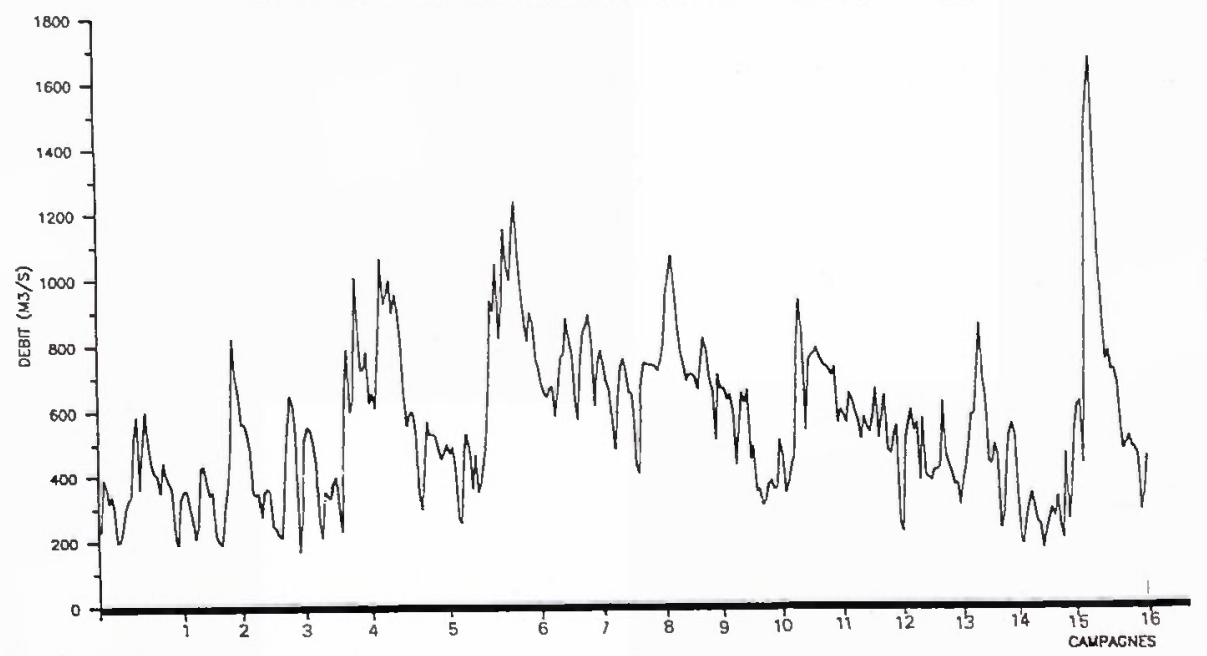

Fig. 3. Débits moyens journaliers relevés dans le Rhône de novembre 1987 à novembre 1988. Les nombres de 1 à 16 localisent les campagnes de mesures dans le temps.

Tableau 2. Caractéristiques physico-chimiques du Rhône (moyenne de l'année d'étude).

\begin{tabular}{|ll|}
\hline $\mathrm{pH}$ & 7,9 \\
conductivité & $293 \mu \mathrm{S} / \mathrm{cm}$ \\
$\mathrm{HCO}_{3}{ }^{-}$ & $196 \mathrm{mg} /$ \\
$\mathrm{SiO}_{2}$ & $2,02 \mathrm{mg} /$ \\
$\mathrm{SiCl}^{-}$ & $6,8 \mathrm{mg} /$ \\
$\mathrm{SO}_{4}-$ & $42,4 \mathrm{mg} /$ \\
$\mathrm{NH}_{4}{ }^{-}$ & $0,17 \mathrm{mg} /$ \\
$\mathrm{NO}_{2}{ }^{-}$ & $0,10 \mathrm{mg} /$ \\
$\mathrm{NO}_{3}{ }^{-}$ & $3,14 \mathrm{mg} /$ \\
$\mathrm{DBO}^{-}$ & $2,7 \mathrm{mg} / \mathrm{O}_{2}$ \\
$\mathrm{PO}_{4}-$ & $0,14 \mathrm{mg} /$ \\
\hline
\end{tabular}

\section{Qualité biologique}

Le Rhône au droit du site du Bugey correspond au niveau typologique B6B7 ou à l'hyporhitron (Verneaux, 1973). La qualité biologique de cette partie du fleuve est moyenne, l'essentiel de la biocénose étant représentée par des organismes détritivores : gammares, Oligochètes, Chironomidae, Hydropsychidae.

\section{Les Gammares}

Ce sont des crustacés Amphipodes. Le genre Gammarus est représenté sur le secteur par deux espèces : $G$. pulex et $G$. fossarum. Leur différenciation repose sur l'examen des antennes des mâles matures qui portent chez $G$. pulex une palette de longues soies au niveau du flagellum. (Karaman, Pinkster, 1977). 


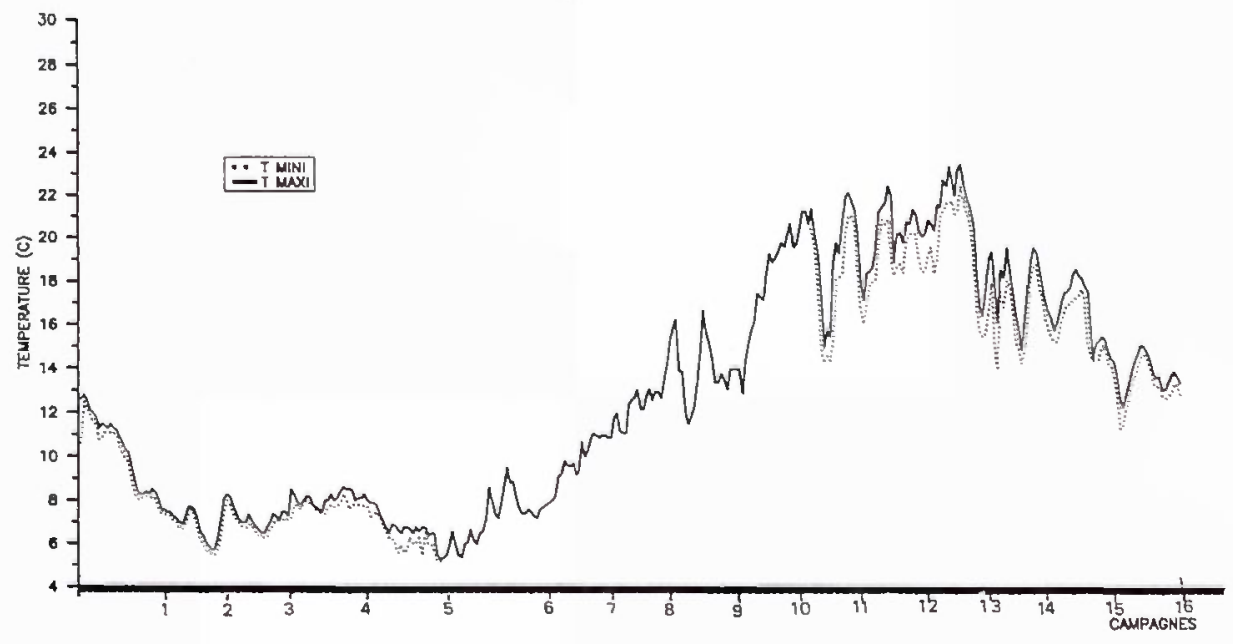

Fig. 4. Températures minimales et maximales journalières relevées à la station amont (1) de novembre 1987 à novembre 1988. Les nombres de 1 à 16 localisent les campagnes de mesures dans le temps.

\section{III.1 Morphologie}

Le corps, aplati latéralement, est formé de 3 parties distinctes : tête, thorax et abdomen, qui portent les appendices :

- 2 paires d'antennes multiarticulées sur la tête;

- 8 segments thoraciques portant une paire de maxillipèdes, 2 paires de gnathopodes, 5 paires de pattes marcheuses, l'ensemble formant les péréiopodes;

- 6 segments abdominaux portant 3 paires de pléopodes et 3 paires d'uropodes.

Le corps se termine par le telson.

\section{III.2 Biologie}

Les gammares ont une vie strictement aquatique. Ce sont des organismes ovipares à développement de type direct, les œufs se développent dans une poche incubatrice : le marsupium.

Leur croissance est de type linéaire exponentiel (Iversen et Jessen, 1977; Sutcliffe et al., 1981; Mortenson, 1982), l'augmentation de taille s'effectue lors des mues, pendant toute la durée de vie des individus.

De nombreux auteurs (Roux, 1970, 1975; Welton et Clarke, 1980; Sarvivo, 1980; Sutcliffe et al., 1981b; Nilsson et Sjöström, 1977; Gee, 1988) ont montré que la reproduction, la croissance et la durée de vie sont dépendantes des conditions du milieu (température, photopériode, disponibilité en nourriture...).

L'influence du facteur température a été mise en évidence par de nombreux travaux. Teckelmann (1974) a démontré que la température optimale de développement de Gammarus fossarum est de $15^{\circ} \mathrm{C}$. Au delà, la reproduction est perturbée, à $20^{\circ} \mathrm{C}$ la 
maturation des œufs serait bloquée; cette température est également un seuil thermique critique pour les jeunes individus lors de leur première mue.

Ces observations sont confirmées par Welton et Clarke (1980) qui ont établi qu'entre 10 et $15^{\circ} \mathrm{C}$, la reproduction de Gammarus pulex est optimale, et qu'à partir de $20^{\circ} \mathrm{C}$ le nombre de pontes diminue fortement.

Roux C. et Roux A.L. (1967) ont également constaté que : «G. fossarum semble rentrer en léthargie dès $20^{\circ} \mathrm{C}$. Dans la nature, si l'eau des ruisseaux atteint $25^{\circ} \mathrm{C}$, on trouve encore $G$. pulex, on ne trouve plus $G$. fossarum".

D'autre part, Roux A.L., (1971) a montré qu'en laboratoire, la température létale pour $G$. fossarum est de $25,8^{\circ}$ C. Barbier et al (1974) don- nent $28,4^{\circ} \mathrm{C}$ comme température létale 50 pour une durée de $96 \mathrm{~h}$ pour les gammares de la Seine acclimatés à $21^{\circ} \mathrm{C}$.

En outre, Franke (1977), dans une étude expérimentale sur le métabolisme respiratoire remarque qu'à partir de $22^{\circ} \mathrm{C}$ G. fossarum ne peut que difficilement accroître son métabolisme et présente des possibilités réduites d'adaptation jusqu'à $25^{\circ} \mathrm{C}$.

\section{Matériel et méthodes}

\section{IV.1 Choix des stations}

Trois stations ont été retenues. Leur substrat est formé de gravier et galets, la vitesse du courant se situe aux environs de $1 \mathrm{~m}^{3} / \mathrm{s}$. Leur emplace-

$$
\begin{aligned}
& 1 \text { : station de référence ; } \\
& 2 \text { : station aval R.D., réchauffée; } \\
& 3 \text { : station aval R.G., peu réchauffée. }
\end{aligned}
$$

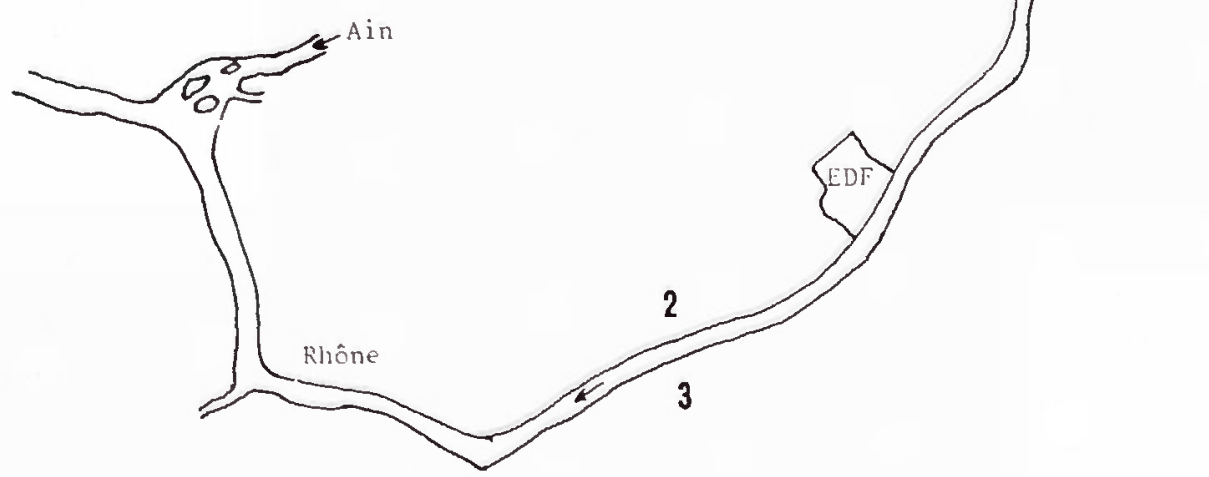

Fig. 5. Situation des points de prélèvement. 
ment est établi en fonction du rejet thermique (Fig. 5).

La station 1 est localisée à $4 \mathrm{~km}$ environ en amont de la centrale. La température de l'eau varie de $5,2^{\circ} \mathrm{C}$ en hiver à $23,5^{\circ} \mathrm{C}$ en été (Fig. 4).

Les stations 2 et 3 sont situées en aval, environ à $5 \mathrm{~km}$ de la centrale.

- en rive droite la station 2 est fortement influencée par le réchauffement (Fig. 2). Les valeurs extrêmes de température sur cette station sont de $8,3^{\circ} \mathrm{C}$ en février, mars et $29,8^{\circ} \mathrm{C}$ en août (Fig. 6).

- en rive gauche la station 3 est soumise à une élévation de température plus faible. En moyenne les températures de cette station sont supérieures de $0,5^{\circ} \mathrm{C}$ à celles de la station de référence.

\section{IV.2 Echantillonnage}

Relevés in situ.

L'échantillonnage des organismes a été réalisé pendant une année (novembre 1987 à novembre 1988) au cours de laquelle 16 campagnes de prélèvement ont été effectuées (tableau 3).

La récolte des organismes a été réalisée à l'aide de substrats artificiels. La colonisation des substrats est assurée par les déplacements volontaires de faible distance des gammares et dans une moindre mesure par la dérive (déplacement des organismes entraînés par le courant à partir de la zone amont) (Nilson et Sjöström, 1977).

Iversen (1977) a montré que la structure de la population en place ne diffère pas significativement de celle

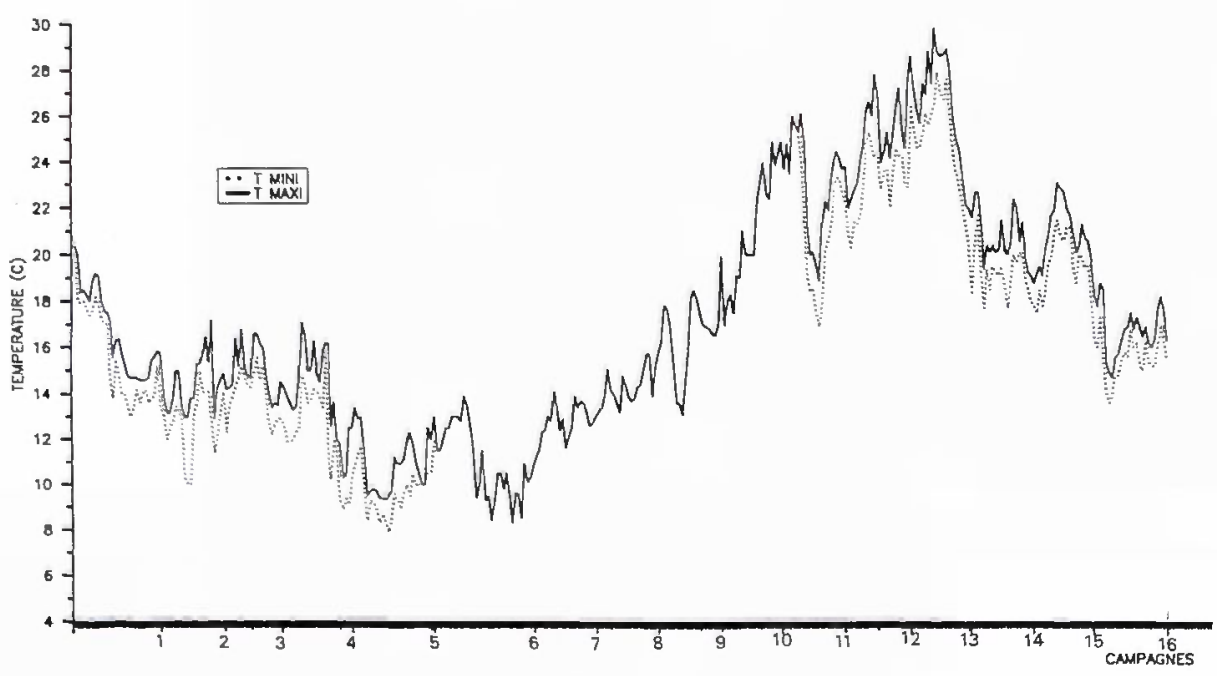

Fig. 6. Températures minimales et maximales journalières relevées à la station aval (2) de novembre 1987 à Novembre 1988. Les nombres de 1 à 16 localisent les campagnes de mesures dans le temps. 
Tableau 3. Calendrier de prélèvements.

\begin{tabular}{|c|c|c|c|c|c|c|c|c|}
\hline $\begin{array}{c}\mathrm{N}^{0} \text { des } \\
\text { campagnes }\end{array}$ & 1 & 2 & 3 & 4 & 5 & 6 & 7 & 8 \\
\hline $\begin{array}{l}\text { Date de départ } \\
\text { Date de retrait }\end{array}$ & $\begin{array}{l}06 / 11 / 87 \\
30 / 11 / 87\end{array}$ & $\begin{array}{l}30 / 11 / 87 \\
21 / 12 / 87\end{array}$ & $\begin{array}{l}21 / 12 / 87 \\
11 / 01 / 88\end{array}$ & $\begin{array}{l}11 / 01 / 88 \\
03 / 02 / 88\end{array}$ & $\begin{array}{l}03 / 02 / 88 \\
01 / 03 / 88\end{array}$ & $\begin{array}{l}01 / 03 / 88 \\
04 / 04 / 88\end{array}$ & $\begin{array}{l}01 / 03 / 88 \\
26 / 04 / 88\end{array}$ & $\begin{array}{l}26 / 04 / 88 \\
16 / 05 / 88\end{array}$ \\
\hline
\end{tabular}

\begin{tabular}{|c|c|c|c|c|c|c|c|c|}
\hline $\begin{array}{c}\mathrm{N}^{0} \text { des } \\
\text { campagnes }\end{array}$ & 9 & 10 & 11 & 12 & 13 & 14 & 15 & 16 \\
\hline $\begin{array}{l}\text { Date de départ } \\
\text { Date de retrait }\end{array}$ & $\begin{array}{c}16 / 05 / 880 \\
6 / 06 / 88\end{array}$ & $\begin{array}{l}06 / 06 / 88 \\
27 / 06 / 88\end{array}$ & $\begin{array}{l}27 / 06 / 88 \\
18 / 07 / 88\end{array}$ & $\begin{array}{l}18 / 07 / 88 \\
18 / 07 / 88\end{array}$ & $\begin{array}{l}08 / 08 / 88 \\
29 / 08 / 88\end{array}$ & $\begin{array}{l}29 / 08 / 88 \\
19 / 09 / 88\end{array}$ & $\begin{array}{l}19 / 09 / 88 \\
10 / 10 / 88\end{array}$ & $\begin{array}{l}10 / 10 / 88 \\
03 / 11 / 88\end{array}$ \\
\hline
\end{tabular}

due à la dérive : la répartition entre les différentes tailles est la même.

Par ailleurs il a également été prouvé que la structure de la population de gammares installée dans un substrat artificiel est liée à la dimension des éléments le composant et évolue dans le temps (Obrdlik, 1972; Pieper, 1978).

Nous avons donc retenu comme type de substrats artificiels ceux utilisés par l'Université de Lyon (Roux et al.; 1976; Khalaf et Tachet, 1978). Les pierres plates préconisées par ces auteurs ont été remplacées par des galets de différentes tailles provenant du Rhône, dont les proportions sont :

$50 \%$ de cailloux fins

$$
(16<\mathrm{D}<32 \mathrm{~mm}) \text {, }
$$

$25 \%$ de cailloux grossiers

$$
(32<\mathrm{D}<64 \mathrm{~mm}) \text {, }
$$

$20 \%$ de petites pierres

$$
(64<\mathrm{D}<96 \mathrm{~mm}) \text {, }
$$

$5 \%$ de pierres moyennes

$(94<D<128 \mathrm{~mm})$.

selon l'échelle ganulométrique de Cummins (1962) et Tolkamp (1980) pour un volume total d'environ $7 \mathrm{dm}^{3}$ d'éléments.
Nilson et Sjöström (1977) ont mis en évidence la dynamique de la colonisation de substrats artificiels. Durant les 8 premiers jours, ce sont surtout les jeunes individus (4 à $5 \mathrm{mg}$ ) qui s'installent, à partir du $17^{\mathrm{e}}$ jour et jusqu'au $32^{\circ}$, toutes les tailles sont représentées et la structure du peuplement reste stable. Au-delà de 32 jours, les auteurs constatent une dominance des individus les plus âgés, due à un phénomène de cannibalisme et de compétition territoriale.

Selon ces observations, nous avons retenu une durée d'immersion de 3 à 4 semaines. Au bout de ce laps de temps les substrats sont relevés et nettoyés afin de récolter l'ensemble de la faune piégée et remis en place; il en résulte donc une présence permanente de substrats sur chaque station pendant la période d'étude.

\section{IV.3 Techniques de laboratoire}

Le prélèvement est filtré par passage à travers une colonne de tamis dont les mailles s'échelonnent de $5 \mathrm{~mm}$ à 
$315 \mu \mathrm{m}$; les gammares sont alors extraits et dénombrés. Ils sont ensuite placés dans un petit tamis dont le fond est divisé en secteurs, ceci afin d'effectuer un sous-échantillonnage permettant d'obtenir entre 150 et 200 individus sur lesquels sont effectuées les mesures.

\section{IV.4 Études préliminaires}

Détermination des espèces.

Dans le Rhône, G. fossarum représente $90 \%$ de la population (Roux, 1970); en amont de Lyon le pourcentage de G. pulex varie de 0 à $4,5 \%$ avec une moyenne de $2,1 \%$ (Dessaix, 1980). Pour le secteur du Bugey, l'examen de 743 individus mâles matures donne $97,2 \%$ de $G$. fossarum et $2,8 \%$ de G. pulex.

Ainsi que l'ont montré les travaux de Roux (1970) ces deux espèces ne présentent pas de décalage dans leur période de reproduction si elles sont soumises aux mêmes conditions écologiques. Ces observations nous ont donc conduit à ne considérer que le genre Gammarus dans son ensemble, sans tenir compte de la présence de 2 espèces.

\section{Mesures}

La longueur des individus a été déterminée à partir de la mesure d'un organe de référence, le carpe du péréiopode 7 (Roux, 1970; Dessaix, 1980).

Pour obtenir des chiffres de production en masse par unité d'échantillonnage il est indispensable d'établir les relations entre :
- la longueur du corps et la longueur du carpe de P7,

- la longueur du carpe de P7 et le poids.

\section{Relation taille-longueur P7.}

Cette relation a été établie à partir de mesures préliminaires que nous avons effectuées en janvier 1987 à Saint-Vulbas portant respectivement sur 158 et 126 individus.

Une analyse de covariance a montré que les courbes obtenues pour les 2 ensembles de mesures ne diffèrent pas significativement.

L'équation de la courbe commune (Fig. 7) s'écrit :

$$
\begin{aligned}
& y=9,67 \chi 0,796 \\
& y=\text { longueur du corps, } \\
& \chi=\text { longueur du carpe P7 } \\
& \text { avec } r=0,979 .
\end{aligned}
$$

\section{Relation poids frais-longueur de P7.}

La pesée a été effectuée sur l'ensemble des 2 lots précédents. On obtient la relation suivante (Fig. 8) :

$\log _{10} P=2,436 \log _{10} L+1,173$

avec $r=0,983$

$P=$ poids frais

$\mathrm{L}=$ Longueur $\mathrm{P} 7$.

\section{IV.5 Estimation de production}

\section{Méthode utilisée}

La production annuelle a été estimée en utilisant la méthode de Hynes et Coleman (1968), corrigée par Hamilton (1969) et complétée par Menzie (1980) à partir de la biomasse 


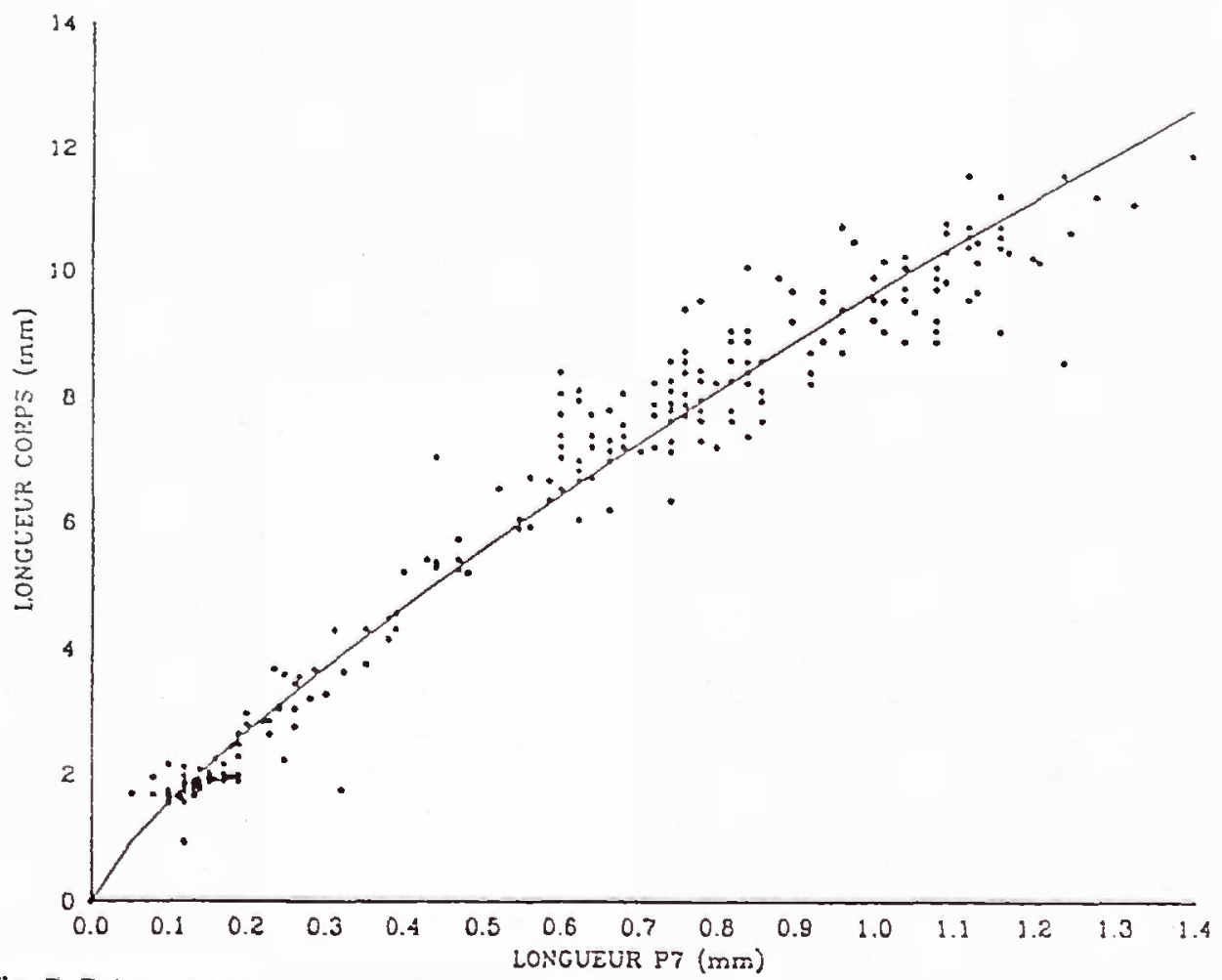

Fig. 7. Relation longueur corps - longueur P7.

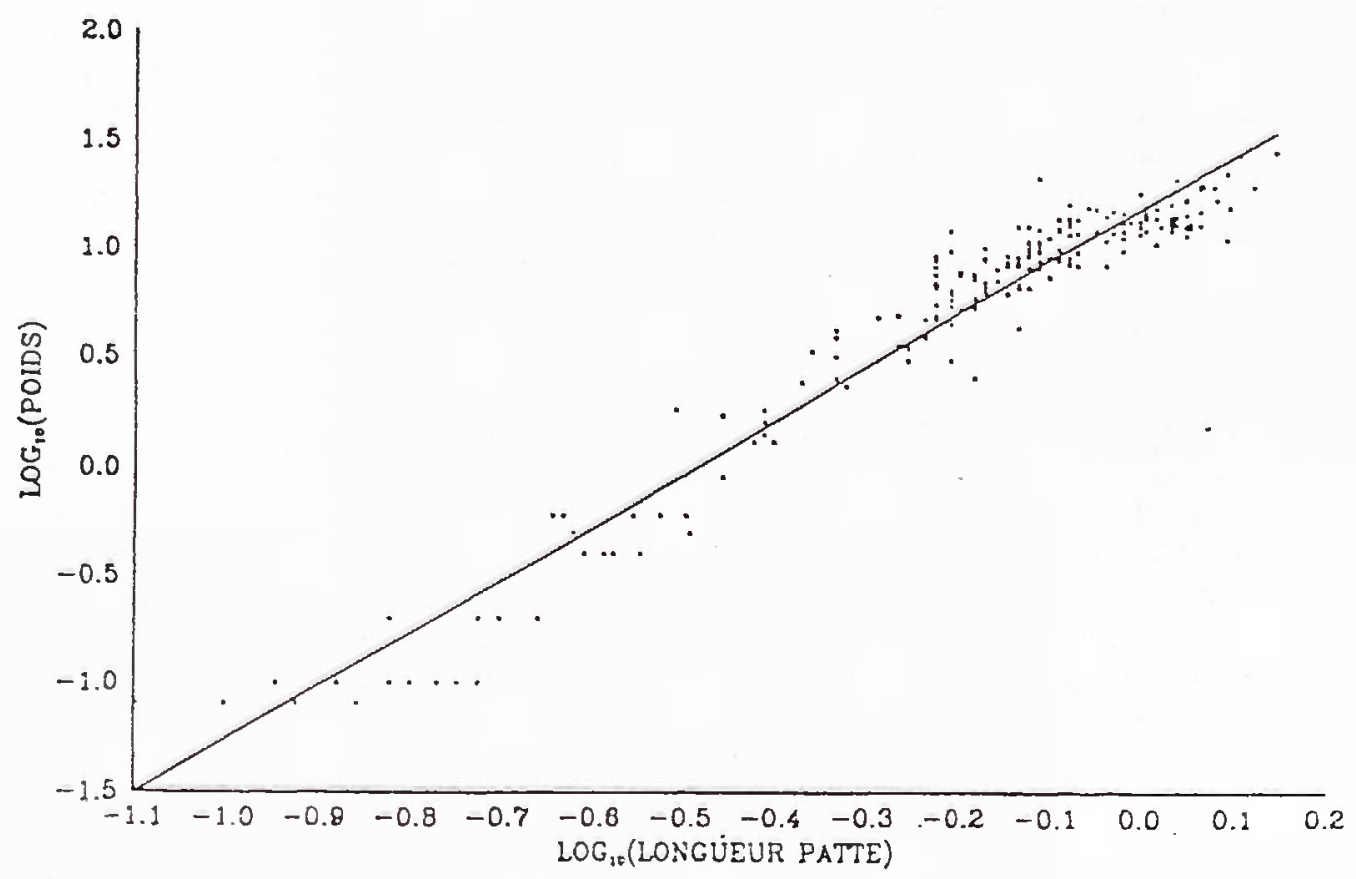

Fig. 8. Relation longueur. P7 - poids frais. 
moyenne de gammares prélevés sur un cycle annuel, répartie par classe de taille, suivant la formule :

$$
\begin{gathered}
P=\left[\sum_{i=1}^{i=n}\left(N_{i}-N_{i-1}\right)\right]\left[W_{i} \times W_{i+1} \times \frac{365}{T}\right] \\
P=\text { production, } \\
n=\text { nombre de classes de taille, } \\
i=\text { classe de taille particulière, } \\
N i=\text { nombre d'individus dans la } \\
\text { classe } i, \\
W i=\text { poids moyen des individus } \\
\text { de la classe } i \\
T=\text { temps moyen, en jours, pour } \\
\text { le passage d'un gammare de la } \\
\text { 1ère à la dernière classe de } \\
\text { taille. }
\end{gathered}
$$

Les travaux de Kruger et Martin (1980) ont permis d'estimer cette production avec des intervalles de confiance à $95 \%$.

\section{Stratégie d'échantillonnage}

L'utilisation de la «méthode des fréquences de taille" pose plusieurs problèmes dont le plus important concerne la variabilité de l'échantillonnage. II nous fallait donc, dans un premier temps, définir certains paramètres pour minimiser la variance de l'estimation de la production afin de rendre plus significatives les différences interstations.

Dans ce but nous avons examiné les résultats d'une campagne d'échantillonnage comportant 10 substrats immergés durant 3 semaines sur la station de référence. Le nombre de gammares récoltés dans ces prélèvements varie de 1950 à 3000 .
Nous avons étudié la relation entre la variance de la production et les 3 données suivantes :

- nombre d'échantillons,

- proportion de l'effectif servant aux mesures (sous-échantillonnage),

- amplitude des classes de taille.

II a résulté de cette analyse qu'avec un nombre d'échantillons égal à quatre par campagne et par station, l'écart-type de l'estimation de la production serait inférieur au dixième de cette production. Quant à l'amplitude des classes de taille, l'optimum se situait entre $0,75 \mathrm{~mm}$ et $1,5 \mathrm{~mm}$. L'amplitude de classe de taille de $1 \mathrm{~mm}$ a été retenue, la classe 1 représente les individus de taille inférieure à $2 \mathrm{~mm}$ et la classe 12 regroupe tous les individus de taille supérieure à $12 \mathrm{~mm}$.

\section{Résultats et interprétation}

\section{V.1 Dynamique des populations}

Effectifs moyens.

La mobilité importante des gammares induit une grande variabilité des effectifs au cours des campagnes (Fig. 9). Cependant, l'allure générale des courbes montre que les effectifs sont relativement peu élevés en hiver (C1 à C5). A partir du mois de mars le nombre d'individus augmente. $\mathrm{Ce}$ phénomène déjà observé par ailleurs (Hynes, 1955; Roux 1970; iversen et Jessen, 1977; Pieper, 1978; Dessaix, $1980 . .$.$) est lié à une reprise de l'ac-$ tivité reproductrice des gammares. Hynes (1955) et Roux (1970) ont 


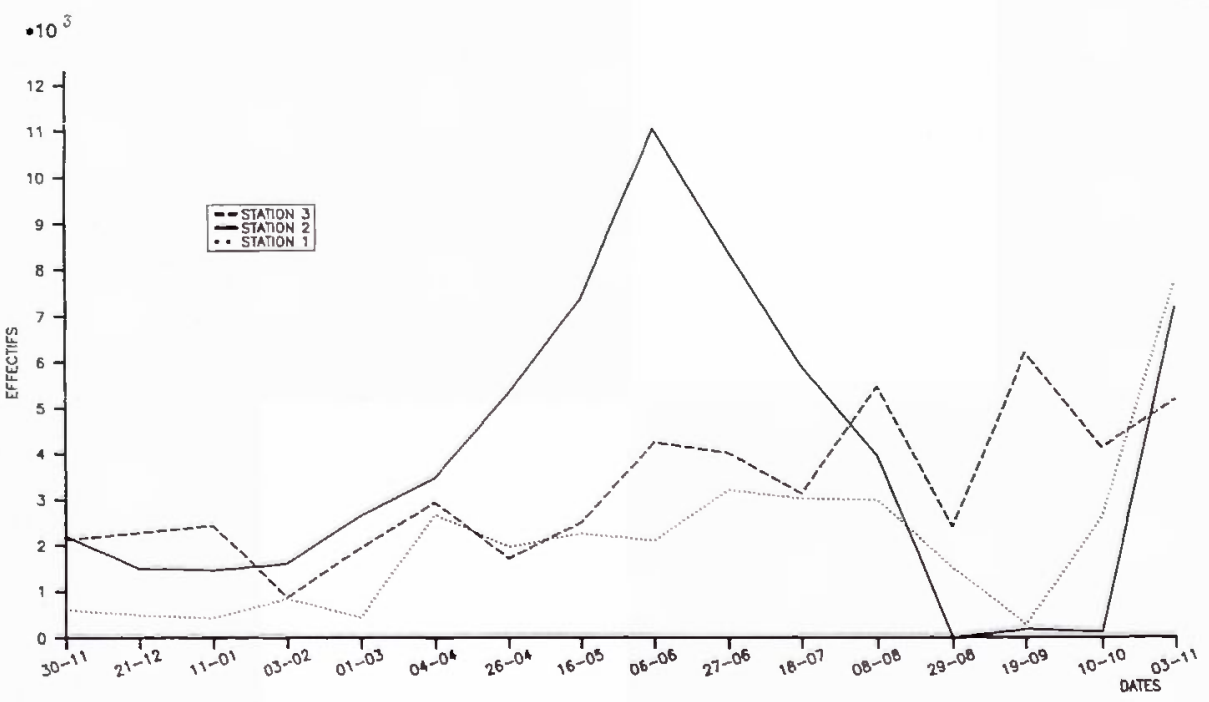

Fig. 9. Evolution des effectifs moyens sur les 3 stations.

montré en particulier qu'une période de repos sexuel débute en automne et s'achève au début de l'hiver, celleci étant déterminée en premier lieu par l'abaissement de température. La reprise de l'activité semble plus liée à un changement de photopériode qu'à une augmentation de température.

Sur les stations 1 et 3 , les effectifs de gammares évoluent de façon comparable mais la station 1 présente généralement des densités inférieures.

Ceci peut être dû à une épaisseur moindre du substrat colonisable par les grammares (gravier, galets), la sous-couche étant de nature marneuse donc inhospitalière. $\mathrm{Par}$ ailleurs, la forte diminution de densité observée à la campagne C14 (septembre) sur cette station est liée à la mise en assec partielle des substrats artificiels par suite d'une période de faible débit.
En revanche, les effectifs moyens du point 2 évoluent différemment : très forte abondance des gammares jusqu'en juin (C9) et disparition totale de ces organismes à la fin du moins d'août (C13), la recolonisation du milieu ne s'effectuant que très lentement (127 individus, récoltés le 10 octobre).

Ce phénomène semble lié aux conditions thermiques particulières qui règnent sur cette station, les autres paramètres du milieu (débit, physico-chimie, photopériode...) étant comparables sur les 3 secteurs.

Durant la période d'avril à début juin (C7 à $\mathrm{C} 9$ ) les températures moyennes de la station 2, varient de $12,9^{\circ} \mathrm{C}$ à $16,7{ }^{\circ} \mathrm{C}$ (tableau 4). Le maximum de $19,9^{\circ} \mathrm{C}$ est atteint le 4 juin. Ces températures sont en moyenne supérieures de $2,5^{\circ} \mathrm{C}$ à celles mesurées sur la station de référence. 
Tableau 4. Évolution des températures du Rhône à l'amont et à l'aval de la centrale du Bugey.

\begin{tabular}{|c|c|c|c|c|c|c|c|c|}
\hline \multirow[b]{2}{*}{ Campagnes } & \multirow{2}{*}{ Stations } & \multirow{2}{*}{\begin{tabular}{|c|} 
Ecart de la \\
température \\
moyenne \\
entre st. 1 et 2 \\
$\left({ }^{\circ} \mathrm{C}\right)$
\end{tabular}} & \multirow{2}{*}{$\begin{array}{l}\text { Température } \\
\text { moyeme } \\
\left.{ }^{\circ} \mathrm{C}\right)\end{array}$} & \multirow[b]{2}{*}{$\begin{array}{l}\text { Température } \\
\text { maximum } \\
\left.{ }^{\circ} \mathrm{C}\right)\end{array}$} & \multicolumn{4}{|c|}{ Nombre de jours* } \\
\hline & & & & & $\begin{array}{c}\text { température } \\
\geq 20^{\circ} \mathrm{C}\end{array}$ & $\begin{array}{l}\text { température } \\
\geq 22,5^{\circ} \mathrm{C}\end{array}$ & $\begin{array}{l}\text { température } \\
\geq 25^{\circ} \mathrm{C}\end{array}$ & $\begin{array}{l}\text { température } \\
\geq 27,5^{\circ} \mathrm{C}\end{array}$ \\
\hline C 7 & $\begin{array}{l}\text { ST.1 } \\
\text { ST.2 }\end{array}$ & 3 & $\begin{array}{r}9,5 \\
12,9\end{array}$ & $\begin{array}{l}11,1 \\
13,9\end{array}$ & & & & \\
\hline $\mathrm{C} 8$ & $\begin{array}{l}\text { ST.1 } \\
\text { ST.2 }\end{array}$ & 1,9 & $\begin{array}{l}12,6 \\
14,5\end{array}$ & $\begin{array}{l}15,3 \\
16,2\end{array}$ & & & & \\
\hline $\mathrm{Cg}$ & $\begin{array}{l}\text { ST.1 } \\
\text { ST.2 }\end{array}$ & 2,6 & $\begin{array}{l}14 \\
16,7\end{array}$ & $\begin{array}{l}16,7 \\
19,9\end{array}$ & & & & \\
\hline $\mathrm{Cro}$ & $\begin{array}{l}\text { ST.1 } \\
\text { ST.2 }\end{array}$ & 4 & $\begin{array}{l}17,6 \\
21,6\end{array}$ & $\begin{array}{l}20,7 \\
24,9\end{array}$ & $\begin{array}{r}2 \\
16\end{array}$ & 9 & & \\
\hline $\mathrm{C} 11$ & $\begin{array}{l}\text { ST.1 } \\
\text { ST.2 }\end{array}$ & 3,3 & $\begin{array}{l}18,9 \\
22,2\end{array}$ & $\begin{array}{l}22,2 \\
25,6\end{array}$ & $\begin{array}{l}12 \\
19\end{array}$ & 12 & 4 & \\
\hline $\mathrm{C} 12$ & $\begin{array}{l}\text { ST.1 } \\
\text { ST.2 }\end{array}$ & 4,8 & $\begin{array}{l}18,8 \\
23,3\end{array}$ & $\begin{array}{l}22,5 \\
28,6\end{array}$ & $\begin{array}{l}14 \\
21\end{array}$ & $\begin{array}{r}1 \\
21\end{array}$ & 11 & 2 \\
\hline G13 & $\begin{array}{l}\text { ST.1 } \\
\text { ST.2 }\end{array}$ & 5 & $\begin{array}{l}20,2 \\
25,3\end{array}$ & $\begin{array}{l}23,5 \\
29,8\end{array}$ & $\begin{array}{l}16 \\
21\end{array}$ & $\begin{array}{r}6 \\
18\end{array}$ & 15 & 7 \\
\hline C14 & $\begin{array}{l}\text { ST.1 } \\
\text { ST.2 }\end{array}$ & 2,7 & $\begin{array}{l}17,9 \\
20,7\end{array}$ & $\begin{array}{l}19,6 \\
22,7\end{array}$ & 7 & & & \\
\hline
\end{tabular}

* Calculé à partir des températures moyennes journalières.

Les températures observées jusqu'au mois de juin à la station 2 coïncidant avec l'augmentation de la durée du jour paraissent donc favoriser le développement et la reproduction des Gammares.

Nos observations sont en accord avec les conclusions des études expérimentales citées précédemment. $\mathrm{La}$ forte diminution des effectifs constatée sur la station 2 , dès le mois de juin, (température moyenne $21,6^{\circ} \mathrm{C}$ ) correspond surtout à la mortalité des très jeunes individus (classe 1).
La baisse des abondances, lors des campagnes suivantes ( $\mathrm{C} 11$ à C13) pendant lesquelles la température moyenne est supérieure à $22^{\circ} \mathrm{C}$ et atteint $25,3^{\circ} \mathrm{C}$ en août (C13), concerne l'ensemble des classes de taille.

De plus les températures élevées atteintes en août : supérieures à $25^{\circ} \mathrm{C}$ pendant 15 jours et à $27^{\circ} \mathrm{C}$ pendant 7 jours, avec un maximum de $29,8^{\circ} \mathrm{C}$ ont entraîné la disparition totale des gammares.

Sur les 2 autres stations, tant que la température moyenne reste infé- 
rieure à $20^{\circ} \mathrm{C}$ (tableau 4) c'est-à-dire jusqu'au 8 août (C12), les effectifs sont relativement stables. Durant le mois d'août, alors que la température dépasse $20^{\circ} \mathrm{C}$ pendant 16 jours et même $22,5{ }^{\circ} \mathrm{C}$ pendant 6 jours, les effectifs diminuent.

\section{Biomasses moyennes - Poids moyens individuels.}

Globalement les biomasses moyennes (Fig. 10) et les effectifs moyens évoluent de façon similaire. La comparaison par station des biomasses et des effectifs permet de suivre la dynamique de la population que l'on peut également caractériser par l'étude de l'évolution temporelle des poids moyens individuels (Fig. 11). Ainsi, les poids individuels élevés en hiver ( $\mathrm{C} 1$ à $\mathrm{C5}$ ) diminuent rapidement entre mars et juin, mettant en évidence une période au cours de la- quelle se produit un fort recrutement. Ils ont ensuite tendance à augmenter et traduisent une diminution de la proportion de jeunes et un grossissement des individus plus marqué en automne. Cette période de l'année correspond généralement à une forte disponibilité en nourriture (chute des feuilles), les gammares accumulant des réserves jusqu'à la fin de l'hiver (Gee 1988).

\section{V.2 Cycle de développement}

\section{Analyse des histogrammes}

L'examen des histogrammes longueur-fréquence établis à partir du pourcentage du nombre total d'individus permet de suivre l'évolution de la population de Gammaridae.

Au niveau de la station 1 (Fig. 12) on observe la présence quasi conti-

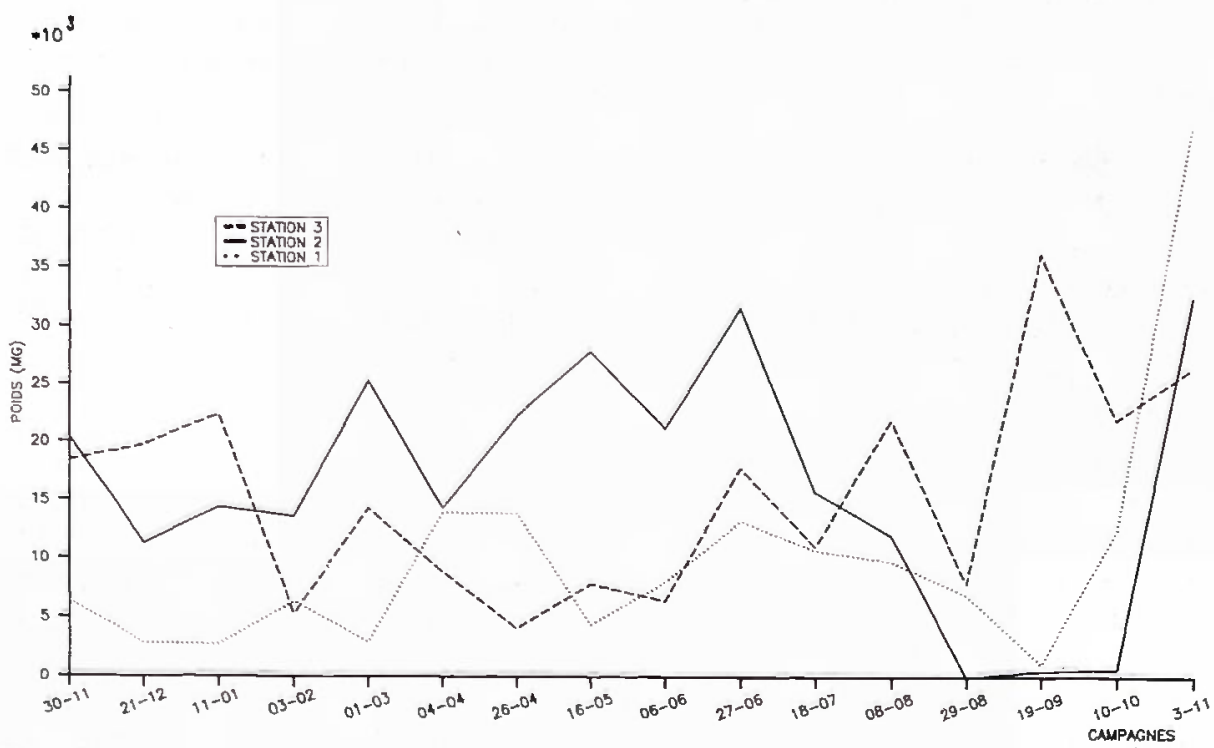

Fig. 10. Evolution des biomasses moyennes sur les 3 stations. 


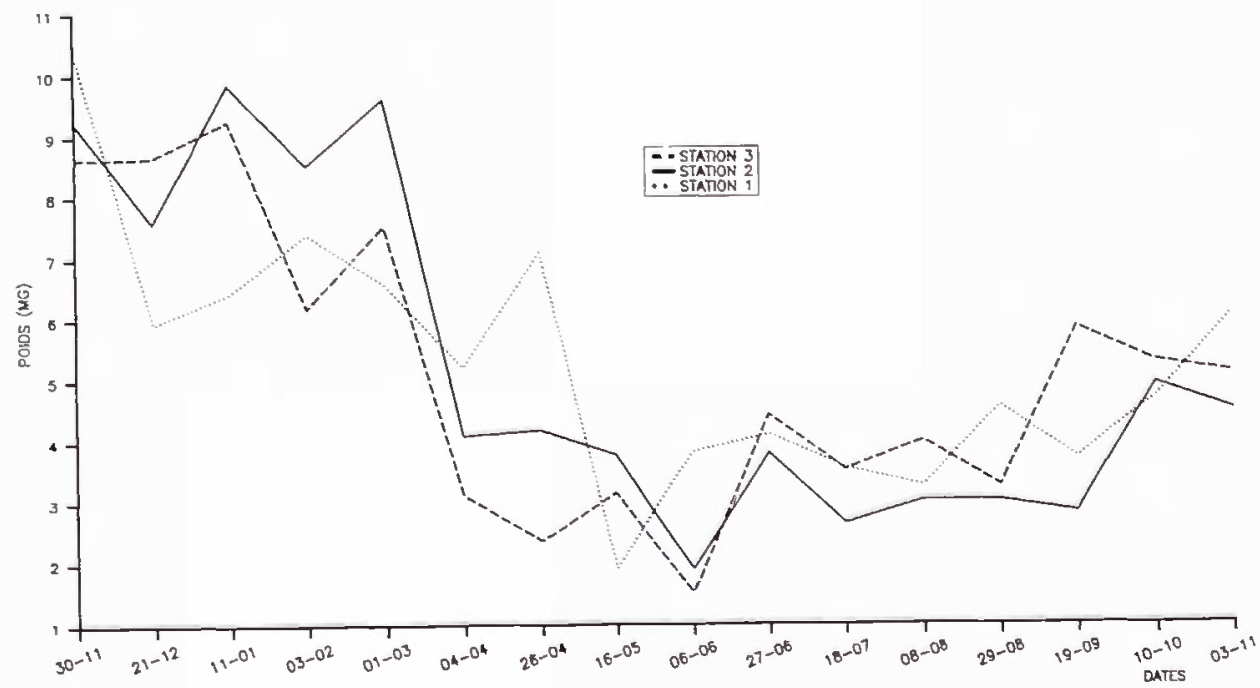

Fig. 11. Evolutions des poids moyens individuels sur les 3 stations.

nue de jeunes individus (classe 1), leur abondance est maximale en mars et avril (C6, C7); elle est encore importante jusqu'en juin (C8, C9). Cette période de l'année correspond à une phase de reproduction intense. Du mois de novembre $(C 1)$ à début février (C4) les histogrammes n'évoluent pas : ce sont les gros individus qui dominent; durant la saison hivernale l'activité de ces organismes est réduite.

La structure de la population de gammares en février (C5) correspond à une transition précédant la période de reproduction.

A partir de juin (C10) et jusqu'en automne on observe sur les histogrammes un déplacement régulier des maxima vers les grandes classes de taille, mettant en évidence la croissance des individus.

Au niveau de la station 2 (Fig. 13) on note une plus forte proportion d'in- dividus de la classe 1 lors des premières campagnes (hiver). Cette classe devient dominante de $\mathrm{C} 6$ à $\mathrm{C} 8$. La forte diminution de l'abondance relative des gammares de grande taille de mars à mai indique que les naissances sont proportionnellement plus importantes qu'à la station amont.

A partir de juin (C10) jusqu'en septembre (C14) le nombre d'individus de la classe 1 est comparativement très faible, il ne dépasse pas $19 \%$. Au mois d'août (C13) alors que la température moyenne journalière a dépassé $28{ }^{\circ} \mathrm{C}$ pendant 7 jours consécutifs, il est intéressant de noter qu'aucun individu (jeune ou adulte) n'a été récolté.

L'évolution de la population de gammares sur la station 3 (Fig. 14) est très comparable à celle de la station de référence. Cependant, les individus de classe 1 sont représentés de façon continue et en plus forte abondance que sur la station 1 . 

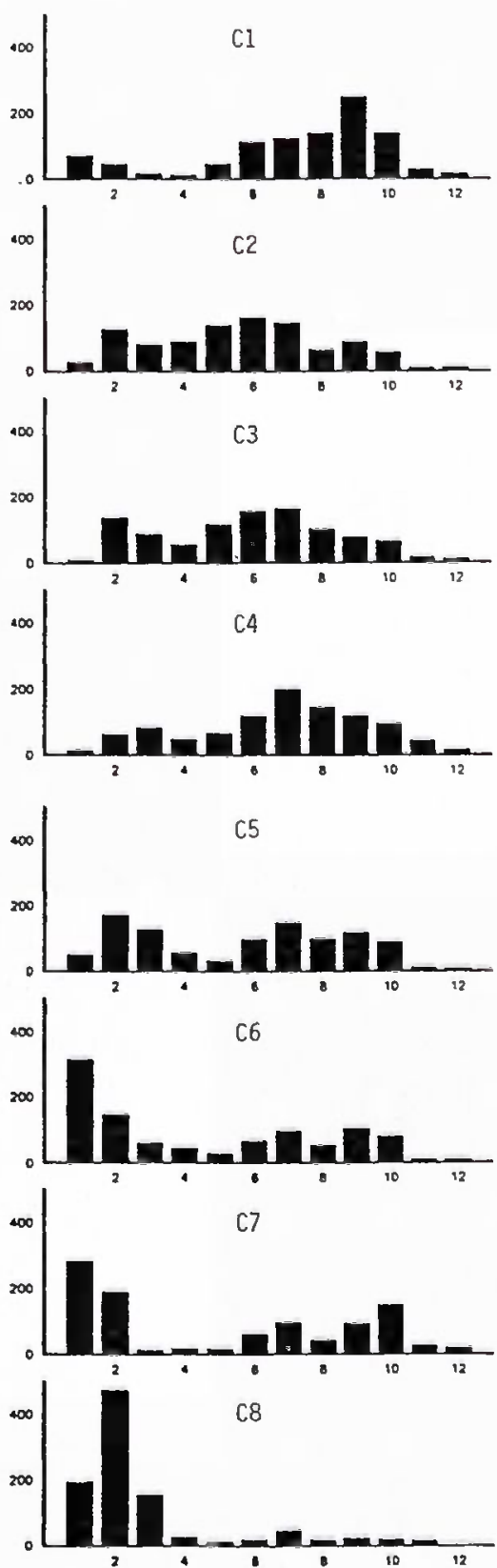
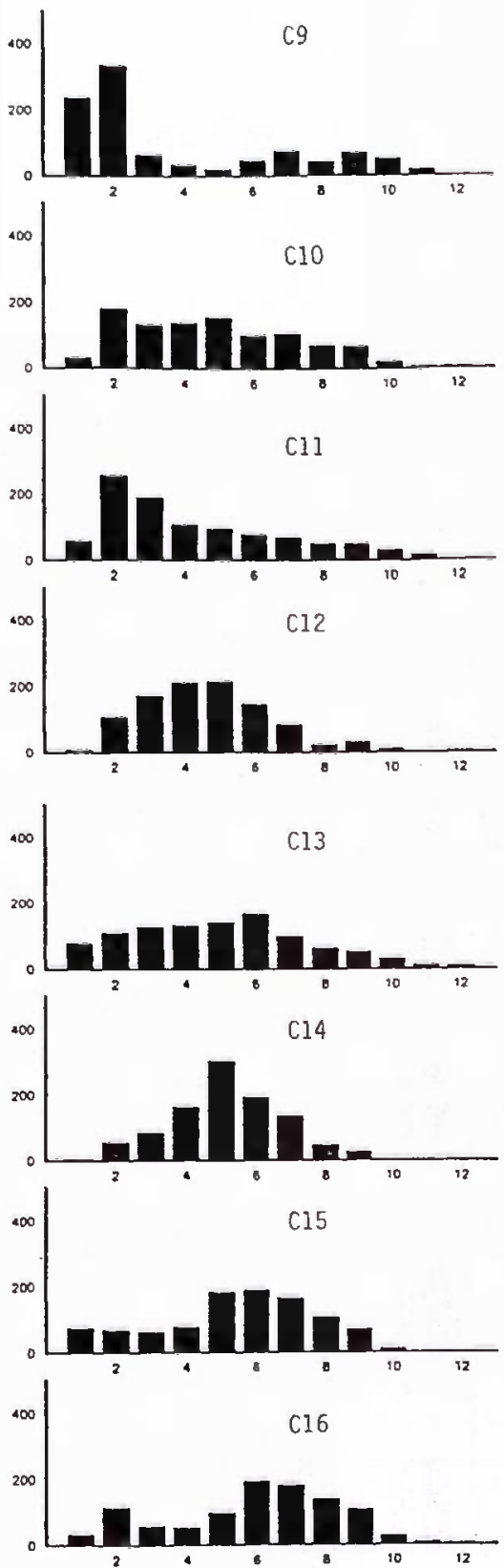

Fig. 12. Station 1 : Répartition des classes de taille $(1 \mathrm{à} 12 \mathrm{~mm})$ en pour mille de l'effectif total des individus récoltés au cours des seize campagnes ( $\mathrm{C} 1$ à $\mathrm{C} 16$ ). 

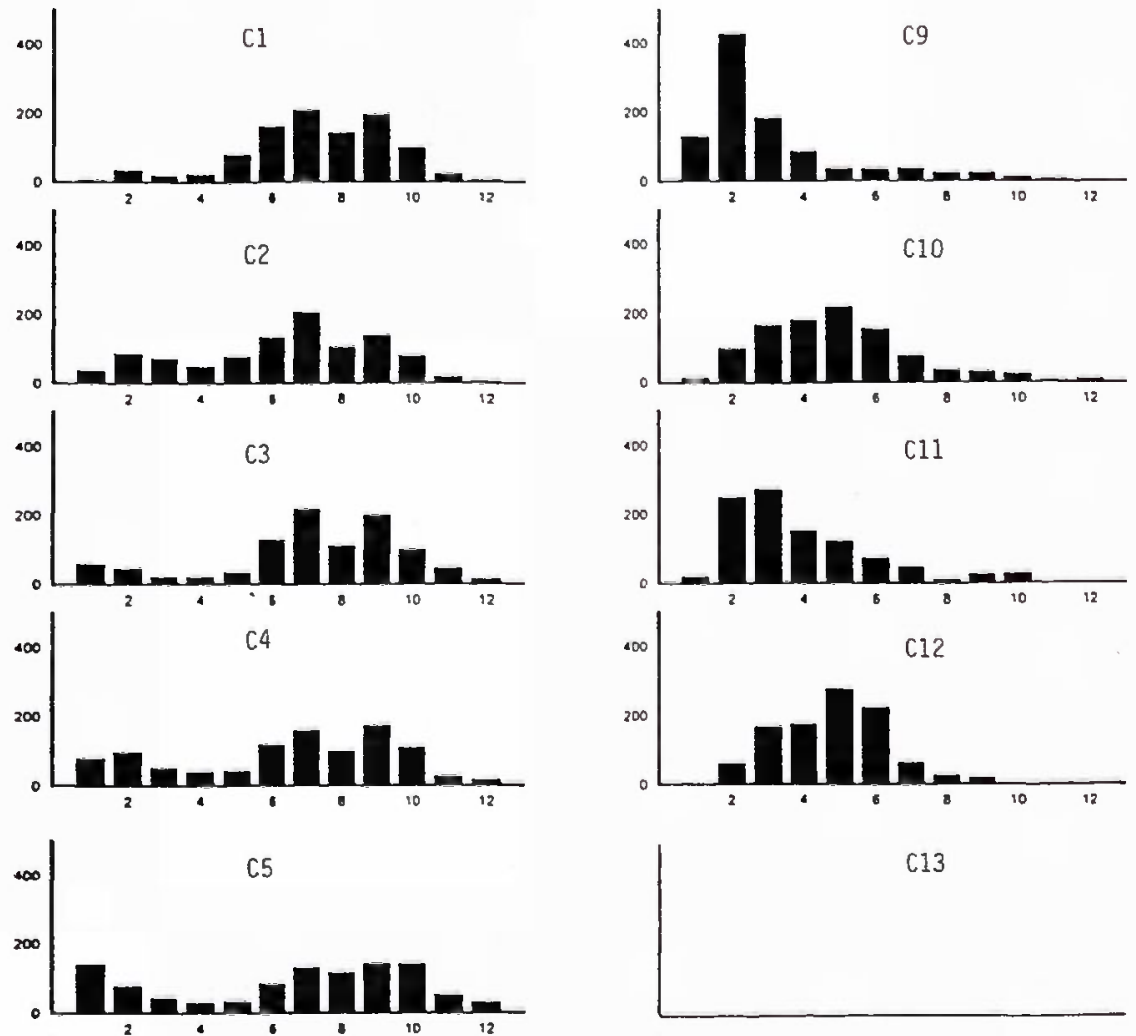

C13
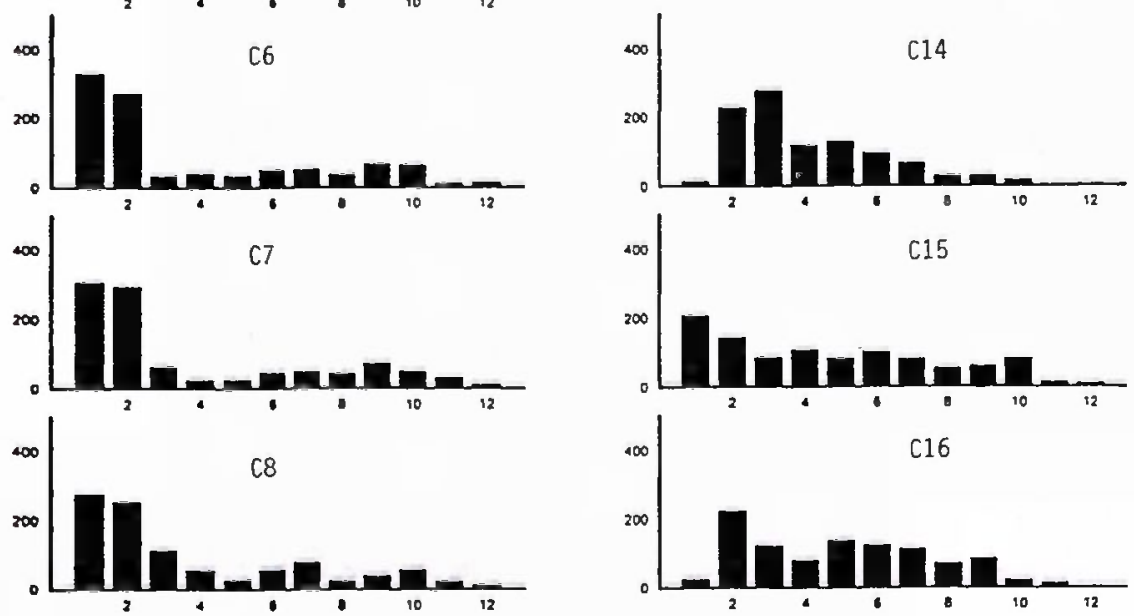

Fig. 13. Station 2 : Répartition des classes de taille $(1$ à $12 \mathrm{~mm}$ ) en pour mille de l'effectif total des individus récoltés au cours des seize campagnes ( $C 1$ à $C 16$ ). 

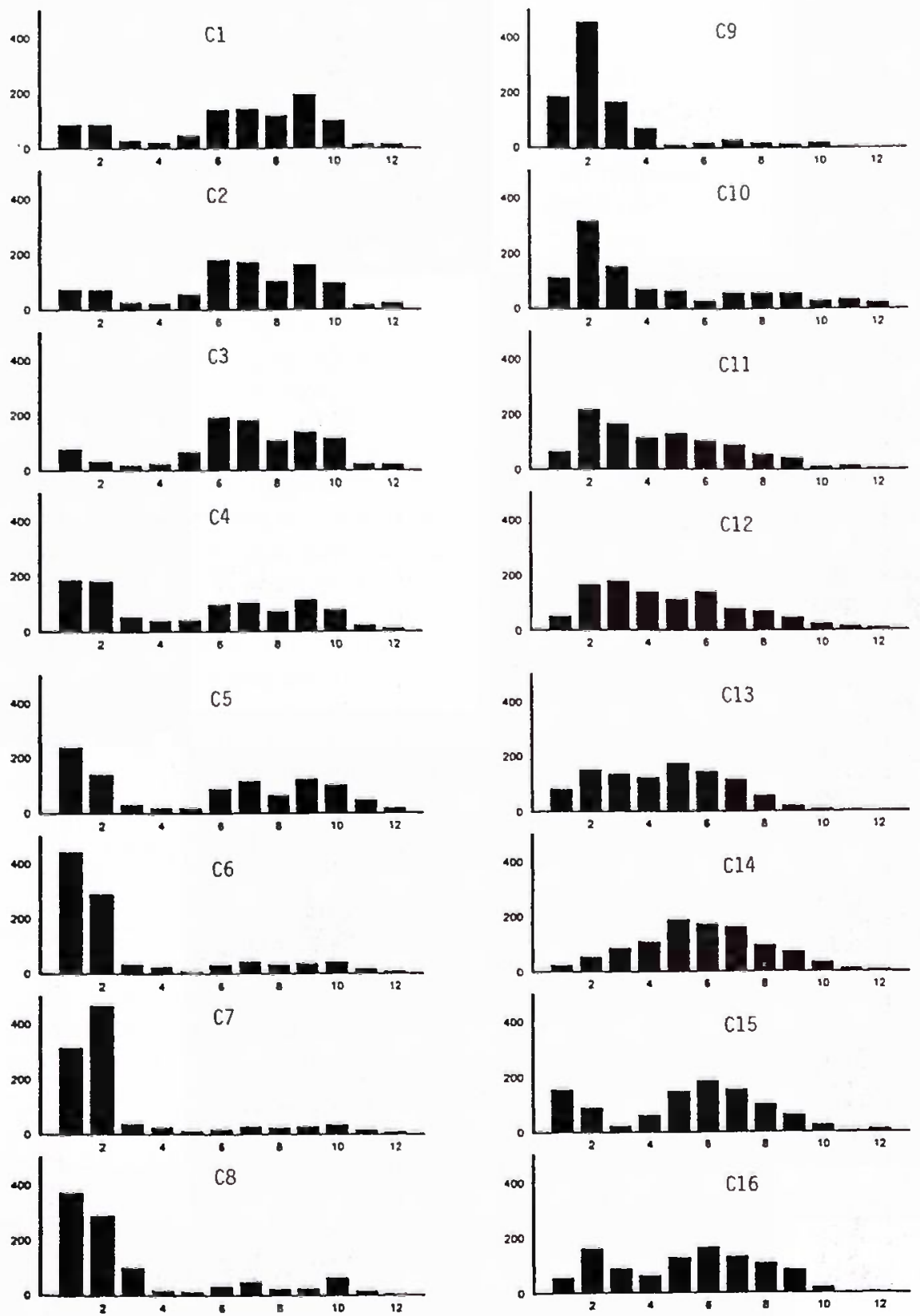

Fig. 14. Station 3: Répartition des classes de taille $(1$ à $12 \mathrm{~mm}$ ) en pour mille de l'effectif total des individus récoltés au cours des seize campagnes (C1 à C16). 


\section{Interprétation}

L'évolution de la structure des populations de gammares intègre à la fois des paramètres physiques (température, débit...) et biologiques (déplacements, colonisation des substrats, phases physiologiques, prédation...) qui rendent délicate une interprétation fiable des histogrammes.

Ceci nous a conduit à traiter les données recueillies par l'analyse factorielle des correspondances (programme ANCORR de l'ADDAD (1) qui permet de mettre en évidence des relations éventuelles entre les modalités des deux variables considérées (taille et date de prélèvements). Ce travail s'est inspiré des recommandations de Chessel et Auda (1982).

L'interprétation des résultats du traitement est effectuée à partir :

- des histogrammes des valeurs propres ou pourcentage d'inertie expliquée par chaque facteur;

- des coordonnées factorielles;

- des contributions des variables aux facteurs.

\section{Histogramme des valeurs propres (tableau 5).}

Sur les stations 1 et 3 , le facteur F1 prédomine nettement sur le facteur F2 : F1 représente respectivement $50,7 \%$ et $55,6 \%$ de l'inertie et F2 $29,6 \%$ et $30,9 \%$.

En revanche sur la station réchauffée (2), ces deux facteurs présentent

(1) Association pour la Diffusion et le Développement de l'analyse des données. des pourcentages d'inertie très voisins $46,2 \%$ et $40,4 \%$.

Sur l'ensemble des 3 stations, ces 2 facteurs représentant plus de $80 \%$ de l'inertie totale ont été les seuls retenus pour l'interprétation.

Etude des variables campagnes d'études.

Pour les stations 1 et 3 , les campagnes $\mathrm{C} 6$ à $\mathrm{C} 9$ apportent les plus fortes contributions à la formation de l'axe F1 (Fig. 15), alors que pour la station 2 elles contribuent à l'axe F2. Pour cette station, le facteur F1 oppose les 7 premières campagnes aux autres, les plus fortes contributions étant apportées par les campagnes $\mathrm{C} 1$ à $\mathrm{C} 5$ et $\mathrm{C} 9$ à $\mathrm{C} 12$. Ces mêmes périodes se trouvent également opposées pour les stations 1 et 3 mais sur F2. Toutefois, leurs contributions à l'axe sont plus faibles pour la station de référence.

Etude des variables classes de taille.

L'analyse des contributions (Fig. 16) montre pour la station 1 que ce sont les classes de taille 1-2 opposées aux classes (4) 5-6 qui contribuent le plus à la formulation de l'axe F1; pour la station 3 , ce sont les classes 1-2 et 5-6 (7). En revanche, pour la station 2, l'axe F1 est essentiellement formé par les classes 34 (5) et (7-8) 9-10. Ce sont ces mêmes groupes de classes de taille qui déterminent la formation de F2 des stations 1 et 3 .

L'interprétation des facteurs en fonction des 2 variables (campagnes 
Tableau 5 : Histogrammes des valeurs propres de la matrice des AFC effectuées sur les données des trois stations.

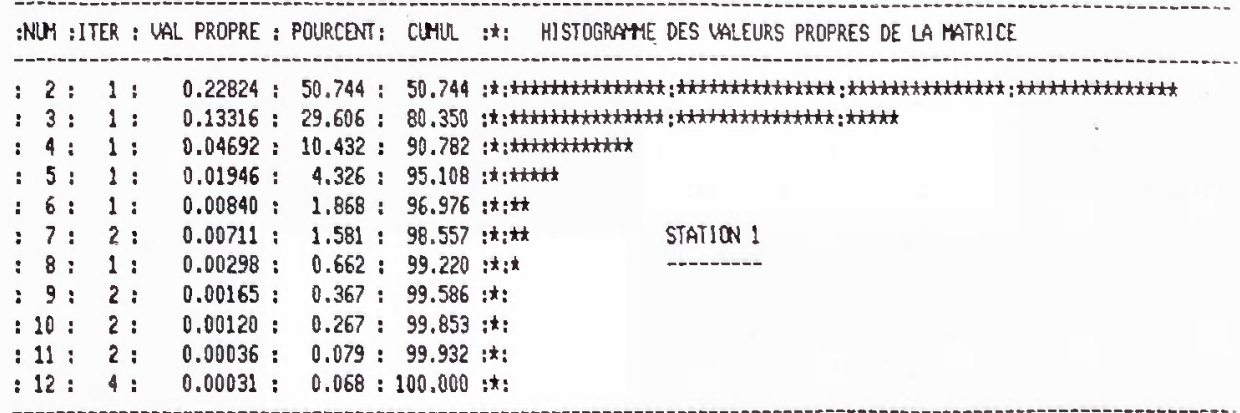

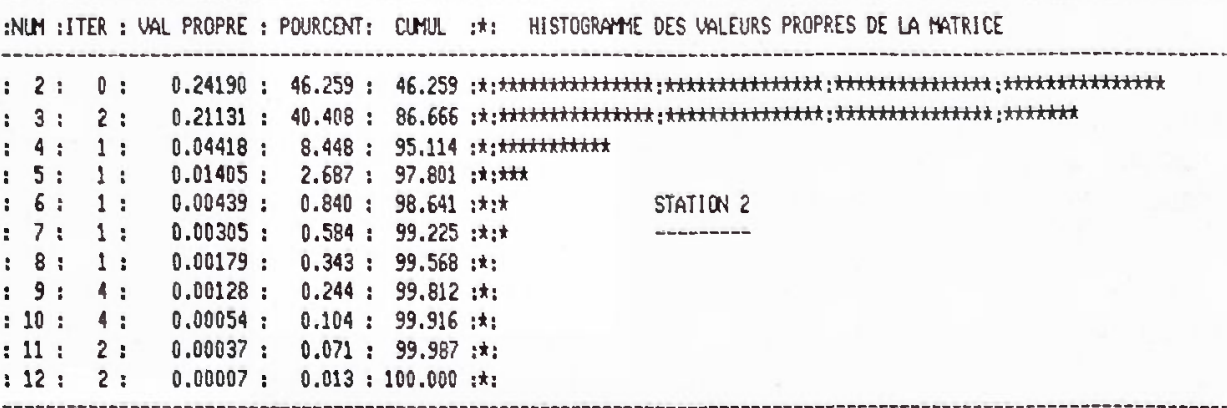

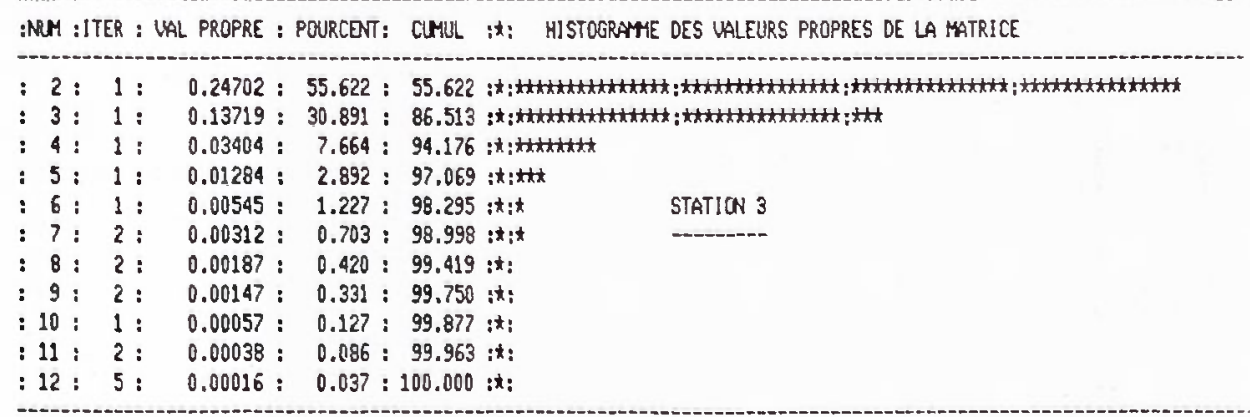

Tableav 5 : Histogrames des valeurs propres de la matrice des AFC effectuées sut les données des trois stations.

et classes de taille) montre que le facteur F2 de la station 2 a la même signification que $\mathrm{F} 1$ des 2 autres points d'étude. Ceci montre que le phénomène qu'ils représentent, c'està-dire l'opposition des classes 1-2 aux classes 5-6 n'est pas prépondérant sur la station réchauffée en raison soit d'un déficit en formes jeunes et/ou d'une croissance plus rapide des individus. 

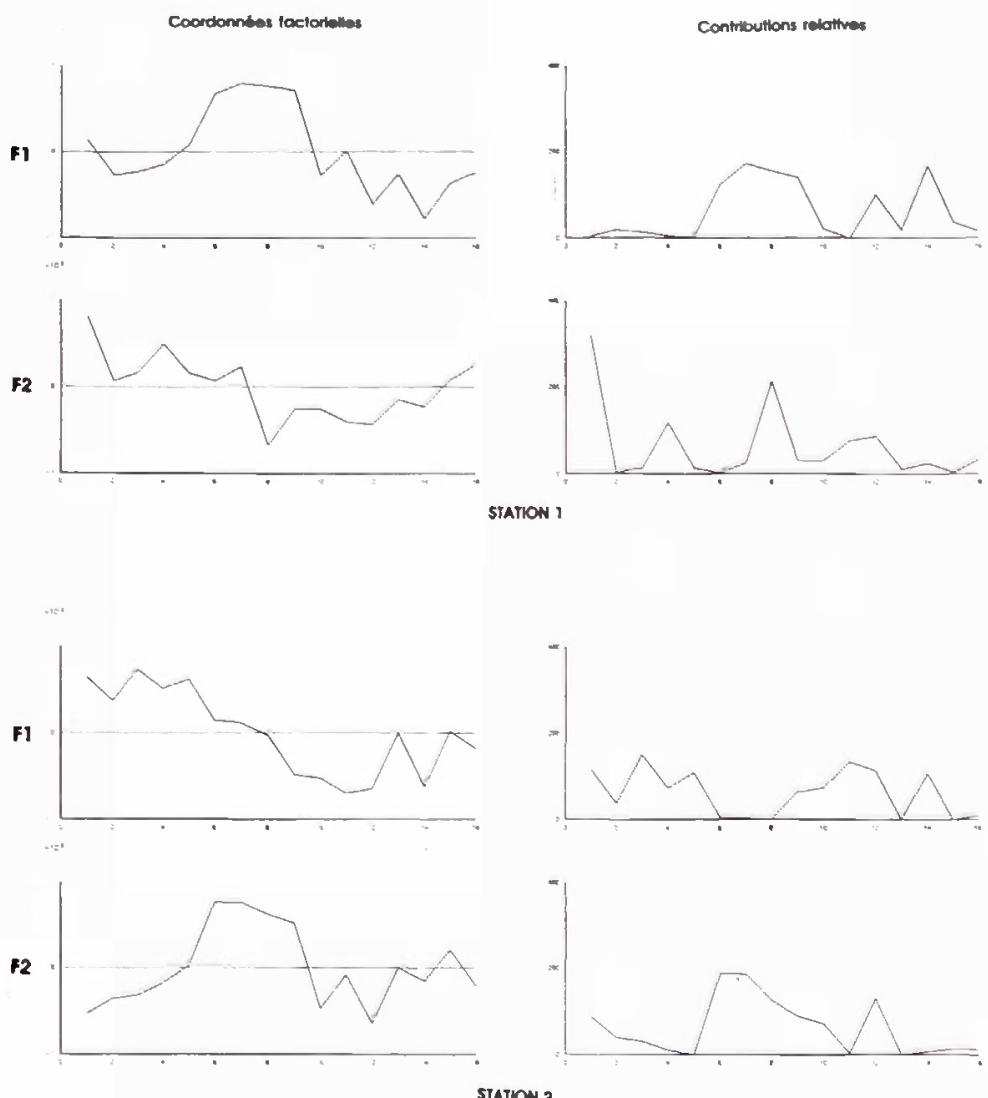

STATION 2
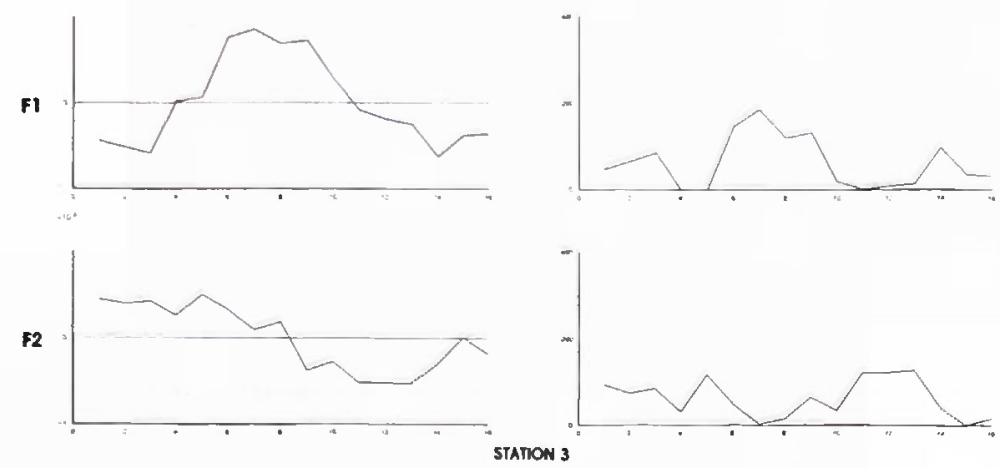

Fig. 15. Interprétation des facteur I \& II en fonction des campagnes d'études. 
Effets de l'échauffement artificiel de l'eau du Rhône

75

F1

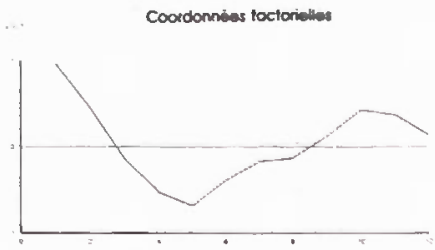

F2

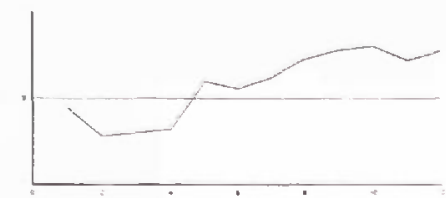

Conidbutions relotives
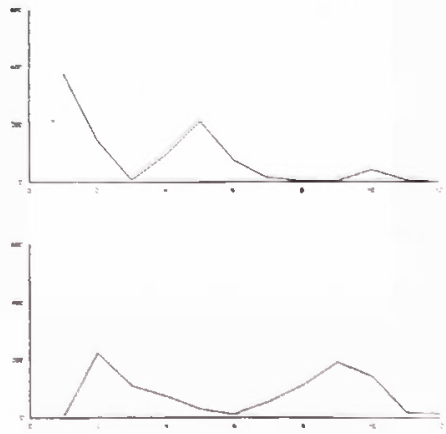

SIATION 1
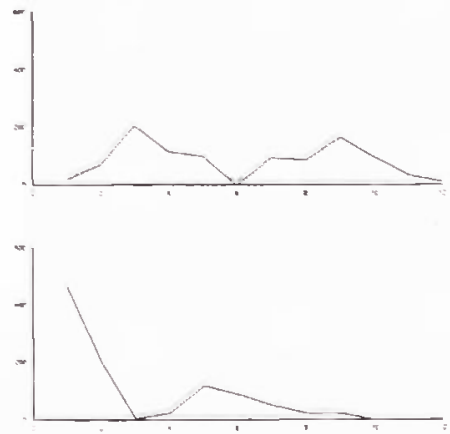

STATION 2

F)
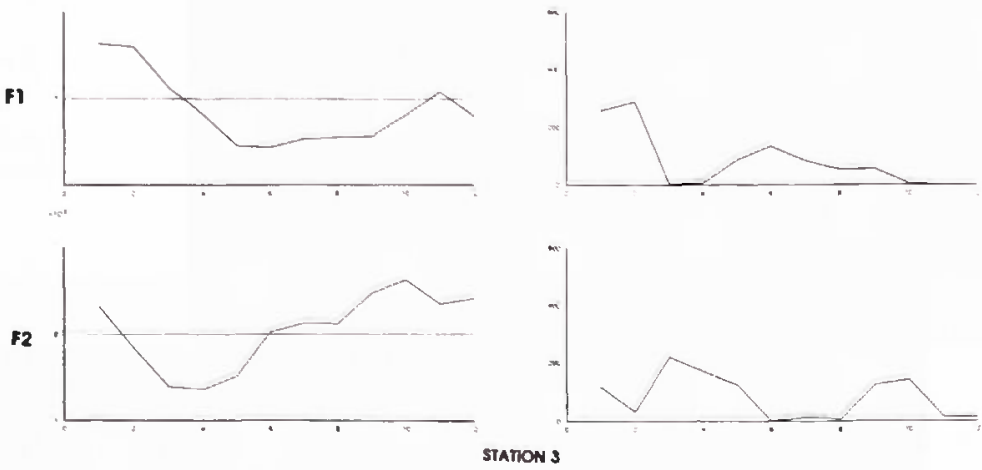

Fig. 16. Interprétation des facteur I \& il en fonction des classes de taille. 
L'étude des 2 facteurs retenus permet de séparer la population de gammares en 4 groupes de classes de taille pertinents pour l'interprétation du cycle de développement :

- les jeunes: classes 1-2

- les juvéniles : classes 3-4

- les jeunes matures :classes 5-6

- les individus âgés : classes 9-10.

Les individus jeunes dominent dans les prélèvement de printemps (C6 à C9); pendant cette période les individus en classe 5-6 sont très peu représentés.

Par ailleurs, les formes âgées (classe 9-10) relativement abondantes en hiver ( $\mathrm{C} 1$ à $\mathrm{C5}$ ) sont progressivement remplacées à partir du mois de juin (C9) par les classes 3-4.

\section{Approche du cycle de développe- ment}

L'ensemble de ces analyses nous ont permis de décrire le cycle de développement des gammares $\mathrm{du}$ Rhône au Bugey.

Ainsi, d'après les histogrammes lissés à partir d'une reconstitution du tableau de contingence avec les seuls facteurs $F 1$ et $F 2$ (Fig. 17, 18, 19) on note que les individus en classe 5 et 6 en hiver (C1 à C4-5) se reproduisent essentiellement au printemps : dominance de classes 1 et 2 de mars à début juin (C6 à $\mathrm{C} 9$ ). La campagne 9 correspond à la fin de la période des naissances intenses. A partir de juin (C10) et jusqu'en octobre (C16), les classes 5 et 6 deviennent progressivement dominantes (phase de croissance) : à ce stade les individus sont capables de se reproduire (Hynes;
1955; Obrdlik, 1972; Dessaix, 1980). Hynes, 1955 a montré que les gammares nés en mars donnaient naissance à une nouvelle génération dès le mois d'août. Dans la présente étude ceci n'apparait pas de façon significative, sans doute en raison d'une température plus élevée pendant l'été, en particulier sur la station 2. Cependant, la présence quasi permanente de très jeunes individus sur les stations 1 et 3 semble confirmer les observations de Hynes. La reproduction de ces organismes s'effectue donc quatre à cinq mois après leur naissance.

D'autre part, les individus, nés en fin de période de reproduction (mai, juin) n'atteignent les classes 5 et 6 qu'au cours de l'hiver, ils ne se reproduiront qu'au printemps suivant, c'est-à-dire 8 à 9 mois après leur naissance.

\section{V.3 Estimation de production.}

La production correspond à la quantité de tissus produite par un organisme par unité de surface et de temps.

L'unité de surface est représentée par un substrat artificiel moyen (moyenne du nombre d'individus récoltés sur les 4 substrats artificiels).

La production est estimée en utilisant les poids moyens par classe de taille et en formulant l'hypothèse que les individus mettent une année pour passer de la classe 1 à la classe 12.

Le taux annuel de renouvellement de la biomasse est évalué à l'aide du rapport $P / B(P=$ production annuelle, $\mathrm{B}=$ biomasse moyenne annuelle). 

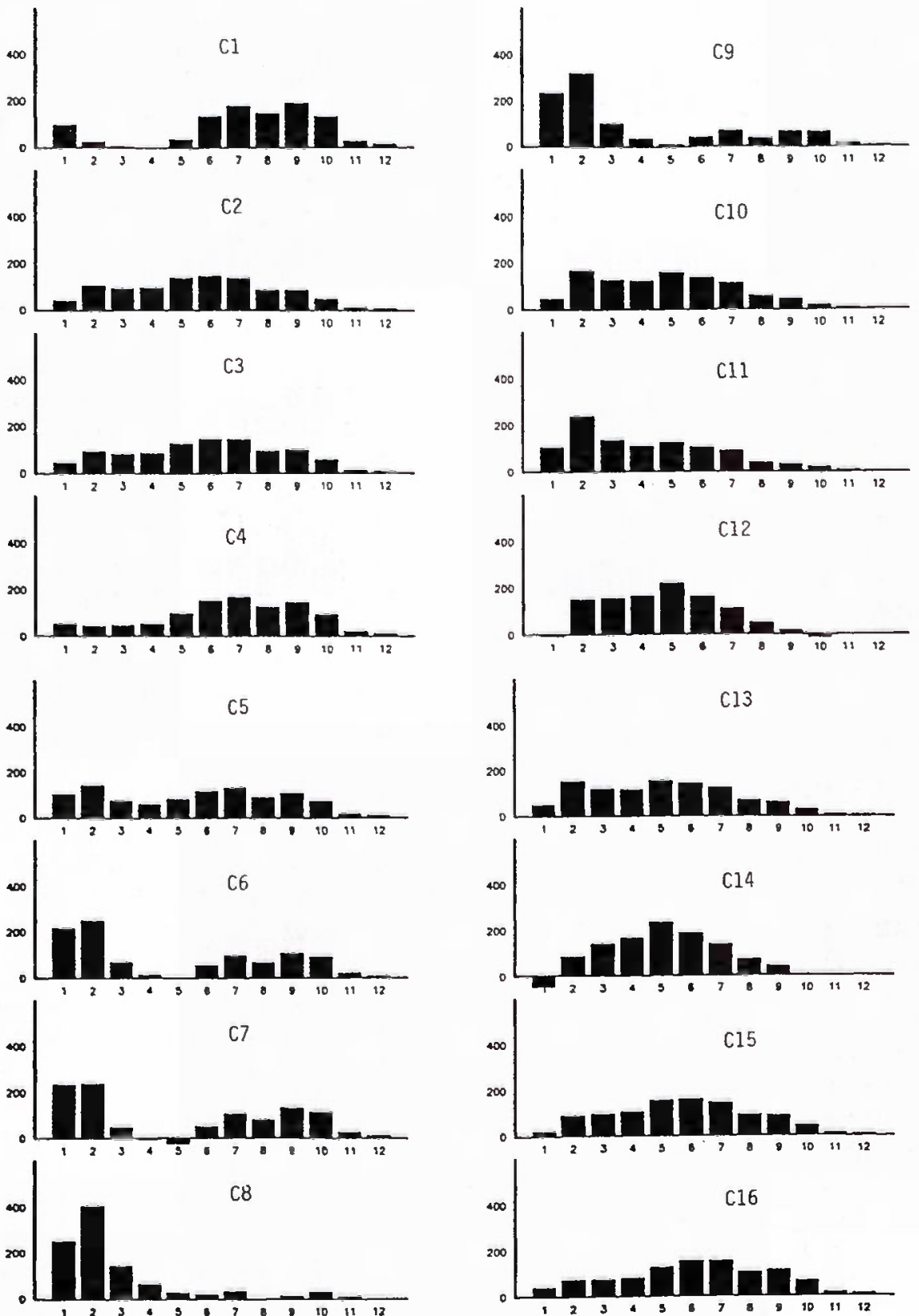

Fig. 17. Station 1 : Répartition des classes de taille $(1$ à $12 \mathrm{~mm}$ ) en pour mille de l'effectif total des individus récoltés au cours des seize campagnes (C1 à $\mathrm{C} 16)$. Histogrammes reconstitués avec les deux premiers facteurs. 

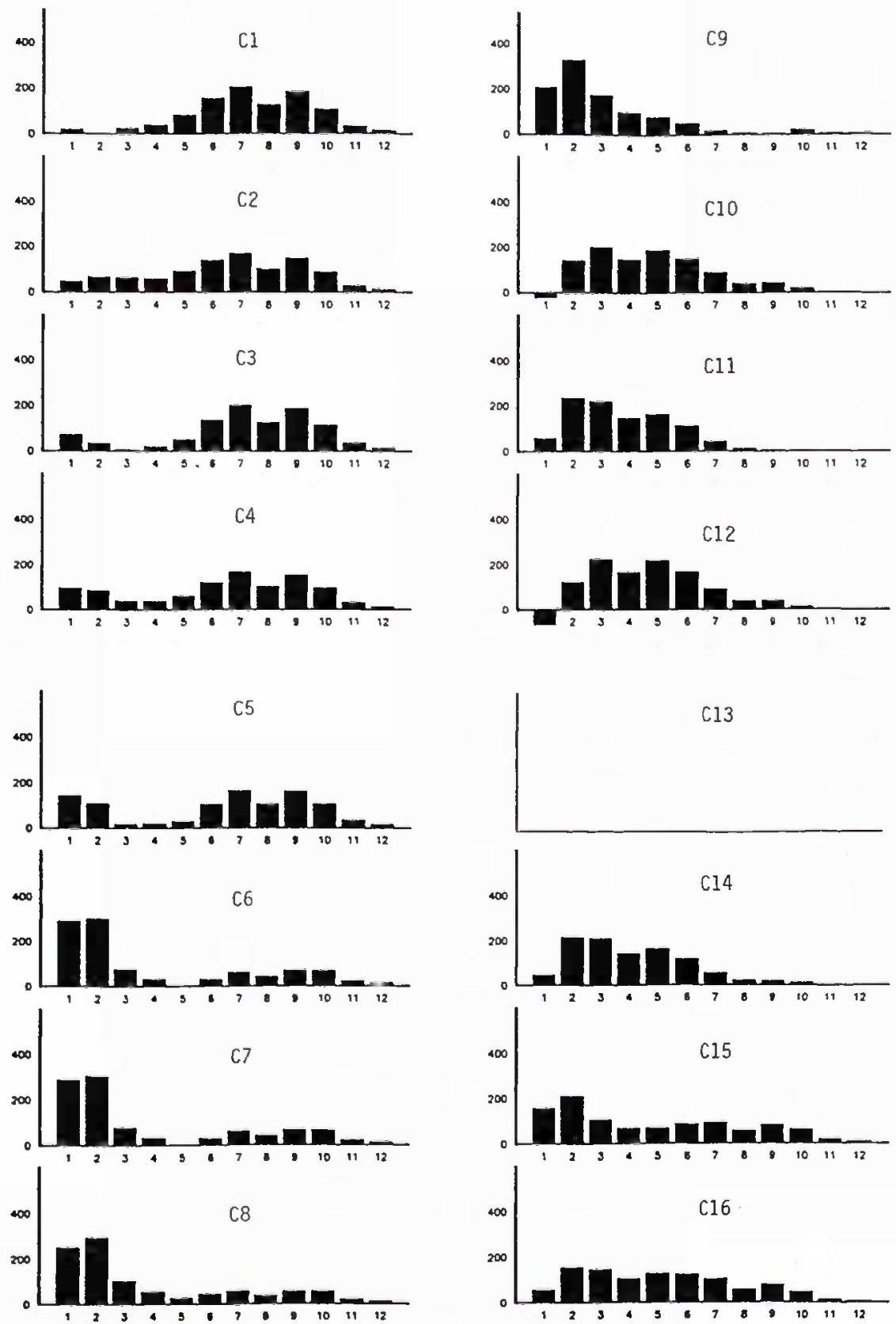

Fig. 18. Station 2 : Répartition des classes de taille $(1$ à $12 \mathrm{~mm})$ en pour mille de l'effectif total des individus récoltés au cours des seize campagnes (C1 à $\mathrm{C} 16)$. Hislogrammes reconstitués avec les deux premiers facteurs. 

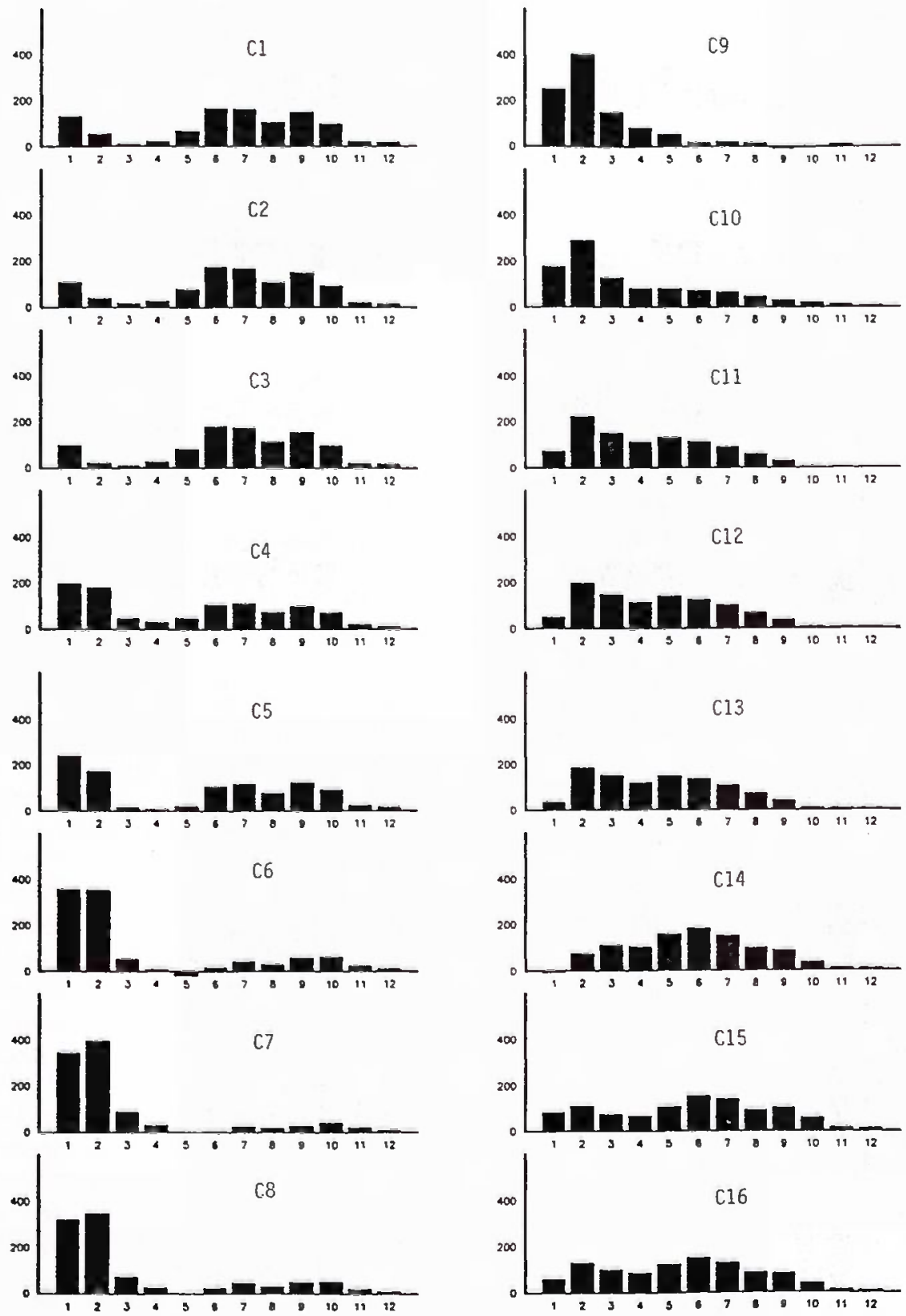

Fig. 19. Station 3 : Répartition des classes de taille $(1$ à $12 \mathrm{~mm})$ en pour mille de l'effectif total des individus récoltés au cours des seize campagnes (C1 à $\mathrm{C16}$ ). Histogrammes reconstitués avec les deux premiers facteurs. 
Les résultats figurent dans le tableau suivant:

Tableau 6. Estimation des productions annuelles.

\begin{tabular}{|c|c|c|c|}
\hline Station & P en g & B en $\mathbf{~}$ & P/B \\
\hline 1 & $41,4 \pm 4$ & 10,20 & 4,09 \\
2 & $71,5 \pm 4$ & 16,44 & 4,35 \\
3 & $66,2 \pm 4$ & 15,62 & 4,24 \\
\hline
\end{tabular}

Les productions annuelles des stations 2 et 3 ne sont pas significativement différentes, elles sont cependant nettement supérieures à celles observées sur la station de référence. Cette observation concorde avec les résultats obtenus à partir des effectifs moyens qui sont généralement les plus faibles à la station 1. Compte tenu de la disparition des gammares en août sur la station 2 , on peut supposer qu'en dehors de l'été la production est nettement supérieure sur ce secteur.

Le calcul du P/B donne des résultats très voisins pour les 3 stations.

Ces valeurs correspondent à celles définies par Waters (1966) qui considère que, pour les invertébrés d'eau douce, dans les zones tempérées, les $P / B$ annuels sont généralement compris entre 2 et 6 .

Dessaix (1980) signale, dans le Rhône en amont de Lyon des taux annuels de renouvellement de biomasse variant de 3,2 à 4 pour les mêmes espèces.

Pour G. pulex, Mortensen (1982) obtient, dans un cours d'eau danois un $P / B$ égal à 2,6 et Waters et Hokenstrom (1980) donnent un $P / B=6$ pour G. pseudolimnaeus.

\section{Conclusion}

Cette étude $\left.{ }^{*}\right)$, réalisée sur 12 mois permet d'obsenver que l'élévation de température de l'eau du Rhône sur le site du Bugey, n'entraîne pas de décalage notable du cycle de développement des gammares. En revanche, elle induit des modifications importantes au niveau de la dynamique des populations. Ainsi, dans la zone réchauffée on note une reproduction plus intense de ces organismes au printemps. Au mois de juin, période à partir de laquelle la température atteint $20^{\circ} \mathrm{C}$, les abondances commencent à diminuer, les individus les plus jeunes disparaissant les premiers. Avec l'augmentation de la température, la diminution de la population s'accentue et au-delà d'une moyenne de $25^{\circ} \mathrm{C}$ (août) les gammares disparaissent totalement du site réchauffé. Dans les deux autres secteurs, lorsque la température dépasse sensiblement $20^{\circ} \mathrm{C}$, on note également une diminution des effectifs. Ces observations sont résumées dans le tableau 7.

(*) Ce travail a été réalisé dans le cadre d'une convention d'étude passée entre EDF. Direction des Etudes et Recherches et le CEMAGREF (E 32/L01 2G 3821). 
Tableau 7. Effets de la température sur le développement des gammares

\begin{tabular}{|c|c|c|c|}
\hline Température & Observations de laboratoire & & $\begin{array}{l}\text { Observations du site } \\
\text { (Rhône-Bugey) }\end{array}$ \\
\hline $15^{\circ} \mathrm{C}$ & $\begin{array}{l}\text { - température optimale de déve- } \\
\text { loppement Teckelmann, } 1974 \\
\text { - reproduction optimale } \\
\text { Welton, Clarke, } 1980 .\end{array}$ & $\begin{array}{c}12,9 \\
\grave{a} \\
16,7^{\circ} \mathrm{C}\end{array}$ & $\begin{array}{l}\text { Naissances abondantes } \\
\text { (station réchautfée) }\end{array}$ \\
\hline $20^{\circ} \mathrm{C}$ & $\begin{array}{l}\text { - Métabolisme ralenti (Roux, } \\
\text { 1967) } \\
\text { - Maturation des ceufs bloquée } \\
\text { (Teckelmann, 1974) } \\
\text { - Diminution du nombre des } \\
\text { pontes (Welton et Clarke, 1980) } \\
\text { - Seuil critique pour la 1re mue } \\
\text { (Teckelmann, 1974) }\end{array}$ & $>20^{\circ} \mathrm{C}$ & $\begin{array}{l}\text { Faible recrutement evou effet } \\
\text { clétals (1) sur les jeunes individus. } \\
\text { (station } 2 \text { campagne } 10 \text { stations } 1 \\
\text { et } 3 \text { campagne 13) }\end{array}$ \\
\hline $22^{\circ} \mathrm{C}$ & $\begin{array}{l}\text { - Seuil critique pour le métabo- } \\
\text { lisme respiratoire (Francke, 1977) }\end{array}$ & $22,5^{\circ} \mathrm{C}$ & Diminution de l'abondance. \\
\hline $\begin{array}{c}24,9 \\
\grave{a} \\
28,4^{\circ} \mathrm{C}\end{array}$ & $\begin{array}{l}\text { - Température létale pour G. fos- } \\
\text { sarum (Roux, 1971) } \\
\text { - température létale pour G. pu- } \\
\text { lex et G. roeslii (Barbier et al.., } \\
\text { 1974) }\end{array}$ & $>25^{\circ} \mathrm{C}$ & $\begin{array}{l}\text { Température "létale" } \\
\text { (station 2) }\end{array}$ \\
\hline
\end{tabular}

(1) Le terme “létal» correspond ici à la disparition des individus.

Un phénomène comparable a été mis en évidence sur les populations des principales espèces de poissons du Rhône qui désertent la zone réchauffée dès que la température dépasse $24^{\circ} \mathrm{C}$.

Les résultats des estimations de production annuelle ne révèlent pas d'effet global du réchauffement, le taux de renouvellement la biomasse (P/B) étant équivalent sur les 3 stations. Cependant, compte-tenu d'une production nulle ou très faible en été sur la station réchauffée, ce résultat implique l'hypothèse qu'au cours de l'année, sans doute au printemps, la production soit nettement supérieure sur ce secteur.

\section{Remerciements}

Les auteurs remercient particulièrement Messieurs F. Trocherie et M. Bouclier pour leur aide précieuse dans l'analyse des données et les traitements informatiques, M. M. Lafont d'avoir bien voulu critiquer le manuscrit et $M^{\text {me }}$ Taillole qui a assuré la dactylographie.

\section{Bibliographie}

Barbier B. \& Champ P. (1974) Résistance des gammares de la Seine aux élévations de température. Bull. Fr. Pisc. $255: 67-71$.

Chessel D., Auda Y. (1982) Lissage d'histogrammes par l'analyse des correspondances. Ronéo. $11 \mathrm{p}$. 
Cummins K.W. (1962) An evaluation of some techniques for the collection and analysis of benthic samples with special emphasis on lotic waters. Am. Midl. Nat. 67 (2) : 477-504.

Dessaix J. (1980) Les Gammares du Rhône en amont de Lyon. Dynamique des populations et estimation de production. Thèse $3^{e}$ cycle. Univ. Lyon. $138 \mathrm{p}$.

Franke U. (1977) Experimentelle Untersuchungen zur Respiration von Gammarus fossarum. Koch 835 (Crustacea Amphipoda) in Abhängigkeit von Temperatur, Sauerstoffkonzentration und Wasserbewegung. Arch. Hydrobiol/Suppl.48 (3/4) : 369-411.

Gee J.H.R. (1988) Population dynamics and morphometrics of Gammarus pulex L. : evidence of seasonal food limitation in a freshwater detritivore. Freshwater Biology 19 : 333-343.

Hamilton A.L. (1969) On estimating annual production. Limnol. Oceanogr. 14 : 771-782.

Hynes M.B.N. (1955) The reproductive cycle of some british freshwater Gammaridae. J. Anim. Ecol. 24 : 352-387.

Hynes M.B.N. Coleman M.J. (1968) A simple method of assessing the annual production of stream benthos. Limnol. Oceanog., 13 (4) : 569-573.

Iversen T.M. Jessen J. (1977) Life cycle drift and production of Gammarus puJex L. (Amphipoda) in a Danish spring. Freshwater Biology, 7 : 287-296.

Karaman G.S. et Pinkster S. (1977) Freshwater Gammarus species from Europe, North Africa and adjacent regions of Asia (Crustacea-Amphipoda). Part 1 : Gammarus pulex group and related species. Bijdragen tot de Dierkunde. 47, $1: 1-97$.

Khalaf G. et Tachet H. (1978) Un problème d'actualité : revue des travaux en matière d'utilisation des substrats artificiels pour l'échantillonnage des macro-invertébrés des eaux courantes. Bull. Ecol. 9 (1) : 29-38.

Krueger C.C. et Martin F.B. (1980) Computation of confidence intervals for the size frequency (Hynes) method of estimating secondary production. Limnol Oceangr. 25 (4) : 773-774.

Menzie C.A. (1980) A note on the Hynes method of estimating secondary production. Limnol. Oceanogr., 24(4) : 770-773.

Mortensen E. (1982) Production of Gammarus pulex $L$. (Amphipoda) in a small danish stream. Hydrobiologia, 87 7782.

Nilsson L.M. (1977) Incubation time, growth and mortality of the amphipod Gammarus pulex under laboratory conditions. Oikos, 29 93-98.

Nilsson L.M. Sjöström P. (1977) Colonization of implanted substrates by differently sized Gammarus pulex (Amphipoda), Oikos, $28: 4348$.

Nisset N. et Verneaux J. (1970) Composantes chimiques des eaux courantes. Discussion et proposition de classes en tant que bases d'interprétation des analyses chimiques. Annls Limnol. 6 52) : 161-190.

Obrdlik P. (1972) A population of Rivulogammarus fossarum Koch (Amphipoda) in a small Fishless stream. Hydrobiologia, 40 (2) : 279-295.

Pieper H.G. (1978) Okophysiologische und produktionsbiologische Untersuchungen and Jugendstadien von Gammarus fossarum Koch 1835. Archiv für Hydrobiologie, Supplementband, 54 : 257-327.

Roux C. et Roux A.L. (1967) Température et métabolisme respiratoire d'espèces sympatriques de Gammares du groupe pulex, (Crustacés, Amphipodes). Annls. Limnol. 3 (1) : 3-16.

Roux A.L. (1970) Le cycle de reproduction de deux espèces étroitement parentes de crustacés Amphipodes: Gammarus 
pulex et Gammarus fossarum. Annls. Limnol. 6 (1) : 27-49.

Roux A.L. (1971) Les Gammares du groupe pulex. Essai de systématique biologique. II. Quelques caractéristiques écologiques et physiologiques. Arch. Zool. exp. gén.. $112:$ 471-503.

Roux A.L. (1975) Température stable et température fluctuante. II. Etude comparative de leurs effets sur la durée d'intermue de Gammaridae femelles. Verh. Internat. Verein. Limnol., $19:$ 3014-3021.

Roux A.L., Tachet H., Neyron M. (1976) Structure et fonctionnement des écosystèmes du Haut-Rhône français. III. Une technique simple et peu onéreuse pour l'étude des macro-invertébrés benthiques des grands fleuves. Bull. Ecol., 7 (4) : 493-496.

Sarvivo V.S. (1980) Temperature aspects of the ecology of the Amphipod Gammarus lacustris Soviet Journal of Ecology. $1: 57-64$.

Sutcliffe D.R.W. and Carrick T.R. (1981a) Number of flagellard segments and moulting in the amphipod Gammarus pulex. Freshwater Biology. 11 : 497 509.

Sutcliffe D.R.W., Carrick T.R. \& Willoughby L.G. (1981b) Effects of diet, body size, age and temperature on growth rates in the amphipod Gammarus pulex. Freshwater Biology. 11 : 183-214.

Teckelmann U. (1974) Temperaturwirkungen auf Wachstum und Stoffwechsel kaltstenothermer Fließwassertiere. Arch. Hydrobiol. 4 : 479-527.

Tolkamp H.H. (1980) Organism Substrate relationships in lowland streams. Agricultural. Research Report 907, Agri. Univ. Wageningen, The Netherlands : $211 \mathrm{pp}$.

Verneaux J. (1973) Cours d'eau de Franche-Comté (Massif du Jura). Recherches écologiques sur le réseau hydrographique du Doubs. Essai de biotypologie. Thèse Doct. Sc. Univ. Besançon, $257 \mathrm{p}$.

Waters T.F. (1966) Production rate, population density and drift of a stream invertebrate. Ecology, 47 (4) : 595-604.

Waters T.F. and Hokenstrom J.C. (1980) Annual production and drift of the stream Amphipod Gammarus pseudolimnaeus in Valley Creek, Minesota. Limnol. Oceanogr., 25 (4); 700-710.

Welton J.S. (1979) Life history and production of the Amphipod Gammarus pulex in a Dorset Chalk stream. Freshwater Biology, $9:$ 263-275.

Welton J.S. \& Clarke R.T. (1980) Laboratory studies on the reproduction and growth of the amphipod, Gammarus pulex. J. Anim. Ecol. 49 : 581-592. 